\title{
Blockchain, business and the fourth industrial revolution: Whence, whither, wherefore and how?
}

Danson Kimani, Kweku Adams, Rexford Attah-Boakye, Subhan Ullah, Jane Frecknall-Hughes, Ja Kim

Accepted for publication in Technological Forecasting and Social Change, Aug 2020

\begin{abstract}
Blockchain is one the most remarkable technological innovations of the 21 st century. The most notable application of blockchain is in the development and operation of cryptocurrencies (e.g. bitcoin, ethereum, among others). Besides the financial services industry, blockchain is also considered in other sectors such as international trade, taxation, supply chain management, business operations and governance. However, blockchain has not been examined comprehensively in all areas of relevant literature. This article conducts a survey of the literature to gain an understanding of the opportunities and issues presented by blockchain in various business functions. The article begins by providing a discussion regarding how the blockchain technology operates. The paper takes a broad focus in its analysis of the prospects of blockchain for various business functions, including banking and the capital markets, corporate governance, international trade, and taxation. The paper demonstrates how organisations and regulators can leverage blockchain to upscale business operations, enhance efficiency and reduce operational costs. The key drawbacks of blockchain that stakeholders need to bear in mind before adopting the technology are also highlighted. The article also reflects on how organisations can tap into blockchain to reap the full potential of the fourth industrial revolution.
\end{abstract}

Keywords: Blockchain, business functions, fourth industrial revolution, technological innovations, fintech 


\section{Introduction}

In the last two decades, the global technological landscape has witnessed an exponential growth of new digital technologies and innovations, or (as per Brennan et al., 2019) 'disruptive ${ }^{1}$ technologies and innovations' (hereinafter 'DTIs'). Such DTIs comprise artificial intelligence (AI), big data, blockchain, cloud computing, and fintech (Cai, 2018; Moll and Yigitbasioglu, 2019). Observers have heralded these developments as the hallmark of the 'fourth industrial revolution'2 (e.g., Schwab, 2016; Nakashima, 2018). Commentators further observe that the digital landscape will continue to evolve rapidly and could look entirely different in the future (Brennan et al., 2019; Lee, 2019). Owing to space constraints, the focus of this paper will be restricted to blockchain as we analyse how this technology is revolutionising the global business landscape. Besides, blockchain is also the underlying technology for several financial innovations including bitcoin, ethereum, and litecoin, among other cryptocurrencies. This paper thus avails readers an appreciation of the technology that underpins many of the innovations that are currently revolutionising the financial services sector around the world.

Blockchain technology works in the form of a distributed ledger system where data used in communication or transactions are stored in a publicly available network of digital blocks (Moll and Yigitbasioglu, 2019). Each such block contains a digital signature and timestamp, which renders the individual blocks virtually immutable (Nakamoto, 2008; Kokina et al., 2017). The digital blocks are arranged together following a complex mathematical logic - a process called 'hashing' (Nakamoto, 2008) - to form a chain of blocks, hence the name blockchain (Angelis and Ribeiro da Silva, 2019; Harris and Wonglimpiyarat, 2019). Compared with shorter blockchains, longer blockchains are argued to be more trustworthy and secure, as they require more effort to be breached by attackers (Nakamoto, 2008; Lai and Lee, 2018).

Blockchain is one of the newest DTIs (Appelbaum and Smith, 2018) and also one not examined in detail in all areas of relevant literature (Islam et al., 2019; Moll and Yigitbasioglu, 2019; Ahluwalia et al., 2020). Blockchain is considered to be one of the most important technological innovations in the 21 st century, with a potential to cut across strategic, value creation and market levels of organisations (e.g., see, Nowiński and Kozma, 2017). Initial research predicts that blockchain technology will disrupt almost all aspects of our lives,

\footnotetext{
${ }^{1}$ Brennan et al. (2019, p. 1, citing from Danneels, 2004, p. 249) defines a "disruptive technology [as] a technology that changes the bases of competition by changing the performance metrics along which firms compete".

${ }^{2}$ This term is used to refer to the transformation of industrial (often manufacturing) and other business processes by the use of 'smart' technology that does not require human intervention.
} 
including financial markets (i.e., securities trading and insurance services) (Brophy, 2019; Singh et al., 2019); business operations and governance (accounting, auditing, supply chain networks) (Bonsón and Bednárová, 2019; Ahluwalia et al., 2020); and healthcare, government operations and public administration (e.g., registration of births and title deeds) (Davidson et al., 2016; Kokina et al., 2017).

The need for a 'trusted third party', such as banks or other central authority is also eliminated by blockchain, thus lowering transaction costs and speeding up business processes and transactions (Gomber et al., 2017; Morkunas et al., 2019). This is also the reason why blockchain technology has been widely embraced by cryptocurrency developers and users (Dai and Vasarhelyi, 2017; Smith and Dhillon, 2019). In addition, blockchain technology has been proposed as a potential solution for fraud prevention in various areas (refer to a survey paper by Li et al. (2020) on the security of blockchain). This includes keeping immutable accounting records about corporate financial transactions, for the benefit of shareholders, tax authorities, and other stakeholders (Wright and De Filippi, 2015; Dai and Vasarhelyi, 2017; Rückeshäuser, 2017; Singh et al., 2019). Blockchain technology can also aid auditors effectively to track and analyse corporate financial transactions in order to reach a more accurate audit opinion (Bonsón and Bednárová, 2019; Moll and Yigitbasioglu, 2019). Finally, the ease with which transactions can be traced within a blockchain system can help to streamline financial market operations (di Castri et al., 2018; Wouda and Opdenakker, 2019), and minimise incidences of money laundering and other unlawful activities (Iansiti and Lakhani, 2017). Generally, in terms of security, it can be concluded that the probability of fraudulent transactions in blockchain is relatively very low compared with other forms of financial technology, such as bitcoin (Goffard, 2019; Phan et al., 2019).

This paper makes several contributions to the literature. First, the paper provides nonspecialist readers with a detailed explanation concerning the workings of blockchain technology. It is hoped that readers, especially those with a non-technical background, will find the paper to be a useful resource in understanding how blockchain functions. Secondly, the paper provides readers with a broader appreciation of blockchain's potential for application in other business areas (i.e., international trade, taxation, supply chain management, business operations and governance), besides the financial services industry. The paper also indicates areas that require further research by scholars. Thirdly, the paper conducts an appraisal of the benefits and drawbacks of blockchain and highlights aspects that policy makers and practitioners should consider in facilitating efficient utilisation of blockchain technology. 
This paper is organised as follows: section 2 critiques prior literature concerning blockchain technology and its application in various business functions. Section 3 presents an explanation of how blockchain works. Section 4 discusses the implications of blockchain for taxation, section 5 discusses the implications of blockchain for the banking industry and capital markets, section 6 discusses the implications of blockchain for corporate governance, section 7 discusses the implications of blockchain for international trade, while section 8 concludes.

\section{Survey of prior literature}

There has been a gradual emergence of published papers on the topic of blockchain and its applications to various business functions and organisational settings. In this section, we present an in-depth review of that literature. This discussion revolves around four themes which have been identified as most salient in understanding the impact of blockchain on business operations, especially in the accounting and finance industry.

\subsection{Regulation of blockchain}

Many governments are noted in the literature as lagging in developing regulations to deal with new technological innovations (Lee and Shin, 2018). This holds back firms which require regulatory approvals before adopting new technologies (Shim and Shin, 2016), or even the adoption of unregulated innovations which might pose hazards in the conduct of business (Leong et al., 2017). Some countries have nonetheless began easing their regulations to facilitate firms seeking to adopt blockchain and other DTIs. For example, Gomber et al. (2018, p. 230) point out Australia, Singapore, Sweden and the UK as some of the countries "where the regulatory and political situations are conducive" to the adoption of DTIs. Singapore, for instance, is noted as the leading country in the world in developing friendly regulatory infrastructure to facilitate the adoption of DTIs (see Ganne, 2018; Lee and Shin, 2018; Wonglimpiyarat, 2018). This has subsequently led to proliferation of various DTIs - including blockchain - which have made it quicker and cheaper for individuals and businesses to settle payments and/or raise capital without needing to go through the traditional banking systems (Zhu and Zhou, 2016; Lee and Shin, 2018; Wonglimpiyarat, 2018). Notably, however, no country is documented in the literature as having developed regulations to pave the way for the integration of blockchain technology in the business sector. Efforts in this regard are led 
primarily by international accounting firms, in the case of the accounting and auditing industry (Karajovic et al., 2019), and non-governmental organisations (NGOs), such as the Accounting Blockchain Coalition (Bonsón and Bednárová, 2019). The discussion above illustrates the key role that regulatory reviews and removal of regulatory constraints can play in facilitating the successful application of blockchain. Blockchain can also be carefully targeted to revolutionise the accounting and finance industries, for instance, to safeguard against fraudulent financial reporting and money laundering which have been areas of debate regarding the adoption of blockchain.

\subsection{Economics of blockchain}

Besides lowering costs and enhancing efficiency in the way business is done (Nowiński and Kozma, 2017), commentators observe that blockchain will also create new ways of "economic organization and governance" (Davidson et al., 2016, p. 8). Davidson et al. (2016) elaborate that blockchain has supplanted the need for a trusted central authority to oversee financial transactions, with that function being delegated (or "decentralised", as in Coyne and McMickle, 2017, pp. 105-110) to users through its distributed ledger system. In this regard, blockchain can serve several functions which are currently a preserve of regulatory (i.e., central banks) and other market mechanisms (i.e., stockbrokers, banks, and other intermediaries), including reducing information asymmetries in the market and replacing costly financial services (Abadi and Brunnermeier, 2018). Berg et al. (2018, p. 7) refer to this as the economics of blockchain, arguing that the "blockchain economy is different from the existing economy", as the prevailing regulatory and market mechanisms will be instantly or gradually made redundant. For instance, the need for auditors to inspect a firm's accounting records would no longer be necessary within a blockchain economy (Karajovic et al., 2019; Schmitz and Leoni, 2019). Similarly, Lee (2019) argues that the widespread application of blockchain in other areas beyond cryptocurrencies (i.e., video games and asset management) heralds blockchain's versatility as a novel economic system in its own right.

\subsection{Blockchain and the fourth industrial revolution}

Although blockchain is still in its nascent stages (see Ahluwalia et al., 2020), observers concur that the technology offers several prospective benefits that will assist businesses to meet the 
demands of the fourth industrial revolution (Moll and Yigitbasioglu, 2019; Singh et al., 2019). Nakashima (2018) argues that blockchain will result in considerable automation of many business functions, which will require little or no human intervention, just as with the newly developed self-driving vehicles. Notwithstanding, this signals an impending job crisis across many industries as many humans might be replaced with faster, cheaper, and more meticulous blockchain technology (Lee and Shin, 2018). Blockchain brings more automation capabilities (i.e., "cyber-physical systems" as per Penas et al., 2017, p. 54), where the technology can manage accounting records and execute financial transactions (Moll and Yigitbasioglu, 2019; Singh et al., 2019), and even resolve any discrepancies using the blockchain consensus algorithms with minimal to no human involvement (Kokina et al., 2017; Ahluwalia et al., 2020). In this respect, blockchain will help organisations to move beyond Industry 3.0 (i.e., enterprise resource planning (ERP) systems which still require considerable human intervention, as per Dai and Vasarhelyi, 2017) into the fourth industrial revolution. Lee (2019, p. 781) also predicts that blockchain will significantly alter, if not replace, many of the current accounting and finance applications, thus heralding a "completely new industrial infrastructure". Finally, Moll and Yigitbasioglu (2019), citing the accountancy and finance industries, suggest that accounting and finance practitioners should not be alarmed by the impeding proliferation of blockchain and related DTIs (e.g., cloud computing, big data and AI), and should instead prepare to harness their superior data gathering and processing power to improve organisational outcomes.

\subsection{Blockchain: prospects and issues moving forward}

The debate concerning the opportunities and challenges presented by blockchain - particularly in the realm of accounting and finance - is still burgeoning. Nevertheless, initial observations concur that blockchain will significantly enhance transparency across accounting and finance, which have also traditionally suffered from low trust due to frequent accounting scandals and misreporting, and financial crimes such as money laundering (e.g., Cai, 2018; Schmitz and Leoni, 2019). In addition, it is predicted that blockchain will offer significant cost efficiencies which will help to reduce frictions in the financial markets (Abadi and Brunnermeier, 2018), eventually passing the cost savings to the market participants (Rozario and Vasarhelyi, 2018). Moreover, developing countries could especially tap into blockchain to spur economic growth and expand financial inclusion (Gonzalez, 2019). 
Conversely, there are some hurdles that will need to be overcome before blockchain can be meaningfully utilised in many industries. Dai and Vasarhelyi (2017), for instance, argue that blockchain is a highly sophisticated technology that requires mastery of information technology (IT) know-how before its effective utilisation by various users, including accountants, managers, auditors, and bankers and even regulators. Inadequately-skilled users may fail to identify vulnerabilities in blockchain systems and could also be easy targets for deleterious entities that may exploit the blockchain system to advance their unlawful activities (Iansiti and Lakhani, 2017). Lastly, the blockchain technology requires significant electrical energy and computer processing capacity (both hardware and software) in order to operate (Ganne, 2018). This could present a barrier to adoption of the technology as the associated costs of operation may outweigh the ensuing benefits (see Zhu and Zhou, 2016; Jamison and Tariq, 2018; Lee, 2019).

\section{How blockchain works}

Blockchain is a database that stores information. Although the current applications of blockchain mostly contain cryptocurrency and business transaction information, any types of information can be stored in a blockchain. For example, other potential applications of blockchain are identity validation, election voting, and personal health information management (Shipman, 2016). As the name suggests, blockchain stores information in the form of blocks, and each block is connected (chained) to the previous block. Blockchains share three common characteristics: a distributed network, peer to peer exchanges, and the use of cryptography and hashes (Lewis, 2018, p. 235). First, unlike other conventional databases where data are stored in a central location and, therefore, are susceptible to a hack, data in blockchains are replicated and stored across multiple nodes (e.g., computers and servers). Because data are stored in multiple locations, it is more difficult for hackers to attack the database, making the blockchain a more secure form of data storage. Secondly, in blockchain, information flows between peers. This is in contrast to a conventional database where information flows between a server and clients. In a blockchain, each node is connected to a number of peer nodes, and these peer nodes are in turn connected to a number of different peer nodes, making up the whole network consisting of connected peer nodes. Each node validates and keeps the record of transactions and communicates its record with others. Based on the consensus protocol of the blockchain, the records of transactions are synchronised to maintain the common record of transactions in the blockchain (Dinh et al., 2018). Therefore, blockchain 
does not rely on a central authority, who/which holds monopolistic power to maintain the database and hence can be corruptible.

The initial application of blockchain technology was bitcoin (Nakamoto, 2008), a public blockchain where anyone can read and participate in transactions. Since then, numerous public blockchains have been developed and are operating. Some applications, however, prefer privacy and control over participation. For example, businesses have proprietary information and want to restrict access to it. In such cases, private blockchain can be developed where a business can control (grant permission to) the participants of the blockchain. A private blockchain can also modify several features of a public blockchain. For example, private blockchain can have a central authority or multiple authorities, use intermediaries, and can develop a trust-based system among participants (Dinh et al., 2018).

To describe how the blockchain works, we use a simple transaction example: Alice wants to pay Bob. The recording of this transaction involves four stages: transaction request, transaction validation, block creation, and ledger update. Figure 1 below demonstrates the process used in blockchain transactions. 


\section{How a blockchain works}

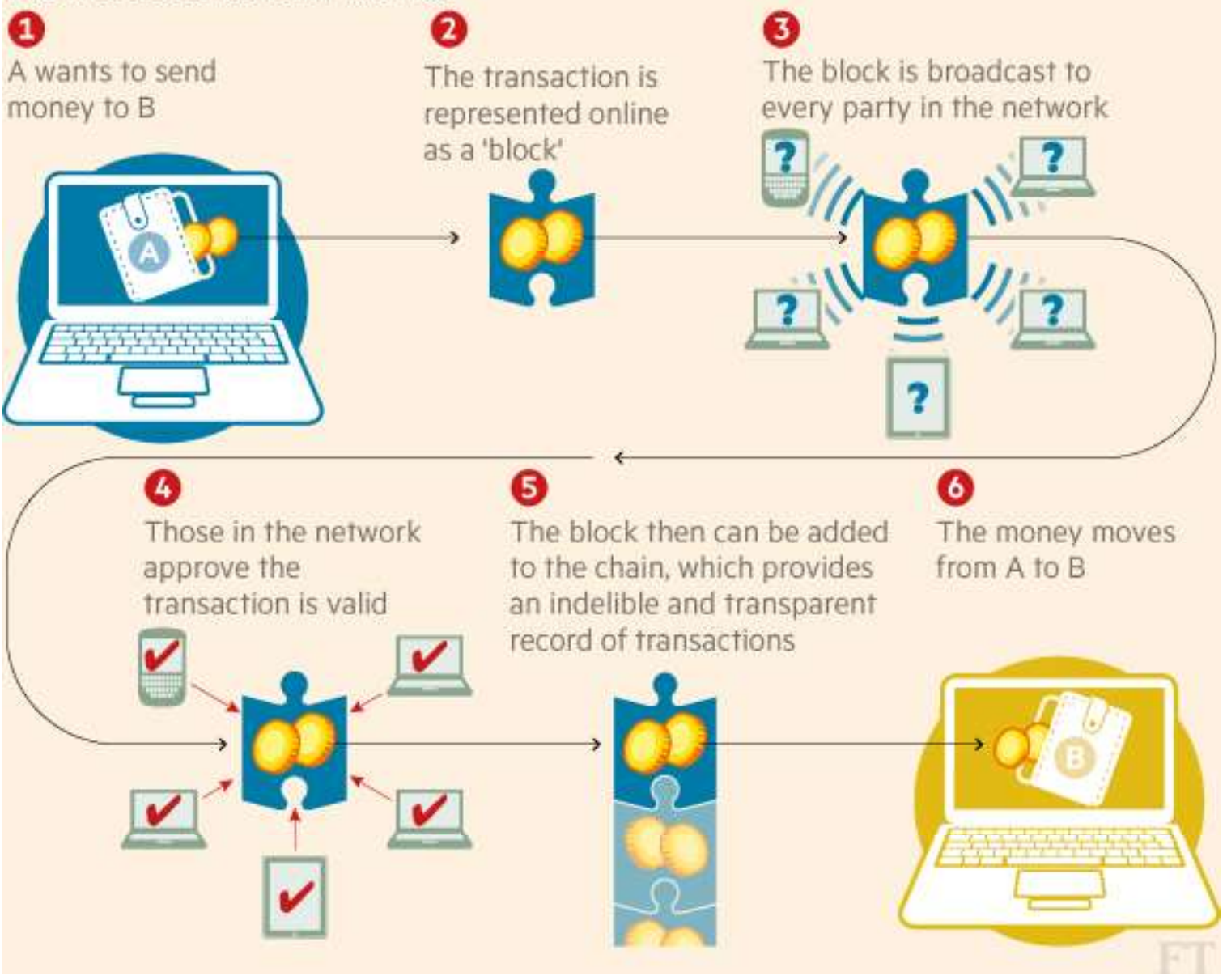

Fig. 1. How blockchain works, from World Economic Forum (2016).

\subsection{Transaction request}

\subsubsection{Types of wallet}

In order to use a blockchain, users (Alice and Bob) need to be connected to blockchain nodes. This is done by using 'wallets' for the blockchain. Wallets allow users to store, send and receive digital currency operating in the blockchain (Vaitkune, 2017). Wallets can be categorised into two types: hot wallet and cold wallet. A hot wallet is an online wallet that is connected to the internet. Users can use internet browsers to access wallet-provider websites, such as Coinbase.com, without downloading any wallet software, or download wallet software on their laptop or mobile phone. A cold wallet, on the other hand, is not connected to the internet. Users can print out their cryptographic keys and addresses in Quick Response (QR) code format on paper or use a hardware wallet that generates cryptographic keys and addresses offline. 
Although cold wallets are less convenient to use, they are considered more secure (Vaitkune, 2017).

\subsubsection{Cryptography: private and public keys}

All digital wallets in blockchain system generate a key pair - private key and public key - and its related address. There are different schemes to generate key pairs. In bitcoin, the Elliptic Curve Digital Signature Algorithm (ECDSA) is used to generate private and public keys (Marshall, 2018). Specifically, for a private key generation, users pick a random number between 0 and $2^{\wedge} 256-1$ (Johnson et al., 2001), which is $115,792,089,237,316,195,423$, 570, 985, 008, 687, 907, 853, 269, 984, 665, 640, 564, 039, 457, 584, 007, 913, 129, 639, 935. In a hexadecimal format, where each character represents four binary digits, bitcoin's private key can be represented as 64 hexadecimal characters ranging from 0 to 9 or $\mathrm{A}$ to $\mathrm{F}$, for example, E9A73D79C6D87DC0FB8A5778633789F44532B3303DA61F10BD67FCD33AA3324B.

Once a private key is chosen, the ECDSA generates a related public key. The private key is considered a master key and is only intended to be known to the holder. On the other hand, the public key is shared in the network so other nodes can use it to validate transactions originated from the related private key (Zheng et al., 2017). The public key is also used to generate an address (the hashed version of the public key) where users can receive digital currencies operating in the blockchain (Asolo, 2019).

\subsubsection{Cryptography: encryption}

In the case of our example where Alice wants to pay Bob, Bob will first generate an address using his public key and share it with Alice. Alice will, in turn, instruct her wallet provider to pay a certain amount of digital currency to Bob's address. Upon Alice's instruction, the wallet provider will create a transaction consisting of a message and a digital signature. A digital signature is a context-specific signature. Therefore, a digital signature is only valid for the message it signs (Kuznetsov et al., 2018). Specifically, the wallet provider generates a digital signature by converting the message into its hash format and encrypting it with Alice's private key. The wallet provider attaches the digital signature to the message and broadcasts them to nodes in the blockchain network (Digital signatures, 2012). 


\subsection{Transaction validation}

\subsubsection{Cryptography: decryption}

Once nodes receive the transaction request, they verify the transaction against the business and technical rules stated in the blockchain protocol (Lewis, 2018). Business rules include the existence of sufficient funds in Alice's address and whether those funds have been spent before. Technical rules check the authenticity of the transaction. Assuming Alice has sufficient funds in her address, nodes validate the authenticity of the transaction using the attached signature in the transaction and the public key. Specifically, nodes decrypt the attached signature with the public key, generating the hashed form of the message the private key originally signed. At the same time, nodes also hash the message in the transaction. If both hash values match, it indicates that the message has been indeed originated from Alice, validating the transaction (Digital signatures, 2012).

\subsubsection{The synchronisation of transactions across nodes}

Once a transaction is validated, it is stored in the Mempool (the abbreviation of 'Memory Pool') of the node. The blockchain software aggregates the validated transactions from the individual Mempools of the nodes and creates the common Mempool of the blockchain, which can be accessed by any nodes (Agrawal, 2019). The validated transactions stay in the Mempool until they are collected by block-creating nodes.

\subsection{Block creation}

Once transactions are validated and stored in the Mempool, block-creating nodes collect these transactions and create a block containing multiple transactions. Blockchain stores data in the unit of digital blocks. Without a central authority who/which processes all the transactions and keeps the record of them in order, blockchain relies on individual nodes to process transactions and keep the record. Therefore, in blockchain, transaction ordering can be inconsistent across nodes. To mitigate the inconsistency in transaction ordering, blockchain uses a block, a group of transactions, to slow down the data entry to the database, allowing nodes to have more time 
to create consensus on the transaction records (Lewis, 2018, pp. 116-119). Different blockchains utilise different block creation times. While frequent block creation allows faster transaction confirmation, it allows less time for nodes to validate blocks and agree on their ordering, creating conflicting blocks in the blockchain. In bitcoin, blocks are generated every ten minutes on average (Frankenfield, 2019a).

\subsubsection{Types of nodes}

In the blockchain, there are different types of nodes performing different functions. Broadly, nodes can be categorised into full nodes and lightweight nodes (Beedham, 2019). Full nodes are active members of the blockchain, storing the whole history of transactions in the blockchain. When articles, including this paper, refer to nodes, they mostly indicate full nodes. Full nodes perform functions of creating, receiving, validating, storing and relaying transactions across nodes. Full nodes also create, receive, validate, store and relay blocks across nodes. As active members, full nodes perform functions essential to the operation of blockchain, including the participation in decision making, such as a change in protocol. Full nodes are expected to download the entire history of transactions (200 gigabytes of data in the case of bitcoin) and run the blockchain software for at least six hours a day (Bitcoin Project, 2020). In the bitcoin network, there are around 10,000 full nodes operating at any given time (Cawrey, 2014; Moos, 2019). Among full nodes, there are also specialised nodes. Blockcreating nodes collect validated transactions from the blockchain Mempool, group them into a block, and create and relay the block to other full nodes. Block-creating nodes are often called mining nodes or miners (in the case of Proof of $\mathrm{Work}^{3}$ ), staking nodes (in the case of Proof of Stake), and authority nodes (in the case of Proof of Authority) (Evans, n.d.). Some blockchains also have master nodes. Unlike block-creating nodes, master nodes do not create blocks. Instead, the main function of master nodes is to validate transactions and to keep the record of transactions (Frankenfield, 2018). Master nodes are expected to be online 24/7, providing stability to the network (Evans, n.d.). For their contributions, master nodes are rewarded with digital currencies. Lightweight nodes, on the other hand, are not active members of the blockchain. Lightweight nodes do not store the entire history of transactions, but only help in authenticating the transactions. Without its own record of blockchain, a lightweight node relies on full nodes for information and broadcasting its transactions (Asolo, 2018).

\footnotetext{
${ }^{3}$ Proof of Work, Proof of Stake and Proof of Authority are algorithms used in the blockchain network.
} 


\subsubsection{Block contents}

A block consists of a block header and block body. A block header, in turn, consists of: (i) the previous block's hash value; (ii) protocol version; (iii) timestamp; (iv) difficulty target; (v) Merkle tree root hash value; and (vi) nonce (Zheng et al., 2017). First, the inclusion of the previous block's hash value in the block header creates a connection between the previous block and the new block. This creates a tamper-resistant database where a change of contents in one block causes changes in hash values in the current and subsequent blocks. If a hacker wants to modify the record of the ledger, he/she needs to create all the current and subsequent blocks while keeping up with the speed of new block creations in the network. This requires the control of at least $51 \%$ of the computing power in the network (Yli-Huumo et al., 2016). Therefore, blockchains are considered immutable. Secondly, the block header includes information about the protocol version, which states block validation rules (Zheng et al., 2017). Thirdly, the block header contains information about the time the block was created. As calculating a hash value for the block requires a timestamp, the timestamp does not reflect the exact time when the block is created. Instead, the timestamp only needs to be accurate within an hour or two (Vaidya, 2016). Fourthly, the difficulty target to calculate a hash value is included in the block header. If hashes are calculated too frequently (or slowly), the blockchain software adjusts the difficulty target to enable slower (or faster) block creation (Dinh et al., 2018). Fifthly, the block header includes the Merkle tree root hash value. A Merkle tree is a hierarchical structure from the bottom level (individual transactions' level) to the top level, using hash functions in each stage (Curran, 2020). Specifically, in the first stage, the hash values of individual transactions are calculated at the bottom level. In the second stage, the hash values of two individual transactions are grouped and hashed. The procedure continues until it reaches the top level where all hash values are summarised into one hash value (i.e., root hash value). The block header includes this root hash value (i.e., Merkle tree root hash value) that summarises all the transactions in the block. Lastly, a nonce (an abbreviation of 'number only used once') is a random number that is used to generate a hash value that is smaller than the difficulty target value (Frankenfield, 2019b). A valid hash value is calculated after multiple trial-and-error attempts. In each attempt, a block-creating node changes nonce until it finds a valid hash value (Nomura Research Institute, 2016).

On the other hand, the block body contains a transaction counter and transaction list. A transaction counter is the count of transactions included in the block. A transaction list is the 
data that blockchain intends to store in its ledger. In each block, however, the first transaction is the reward to the block-creating node, called the 'coinbase' transaction. This is the reward for its work, consisting of the block reward (newly minted digital currencies) and transaction fees (Risius and Spohrer, 2017).

Depending on the blockchain, the block body can also include a smart contract list (YliHuumo et al., 2016; Dinh et al., 2018; Saberi et al., 2019). A smart contract is a digital contract that executes transactions automatically if the specified terms are met, thereby removing intermediaries and human interpretation. For example, an exporter will be paid automatically if the products arrive at the importer's country. Currently, ethereum-based blockchains allow smart contract list (Learn about ethereum, 2020). As smart contracts are digital contracts, they need to be written in computer language for automatic execution.

\subsubsection{Cryptography: hash function}

A hash function is a mathematical function that transforms data of any size into fixed-size data. For example, an MD5 (Message-Digest Algorithm) function generates a 128-bit hash value, while SHA-256 (Secure Hash Algorithm) generates a 256-bit hash value, regardless of whether the input data is a word, sentence, or the entire hard drive (Satoh and Inoue, 2007). A hash function has several characteristics. First, a hash function is deterministic, meaning that the same input data always generate the same hash value. At the same time, it is not feasible to obtain the same hash value from different input data. Secondly, a hash function is a one-way cryptographic function. Therefore, although transforming the input data into a hash value is quick, it is impossible to obtain the original input data from the hash value. Thirdly, a small change in input data generates an extensively different hash value, making it hard to predict a hash value before calculation.

As a result, a hash function allows data validation without knowing the data themselves. If two hash values are identical, it indicates that input data are identical. In a blockchain, a hash value comparison is used when validating the authenticity of transactions (Rosic, n.d.). On the other hand, the difficulty in predicting a hash value makes block-creating nodes conduct multiple trial-and-error calculations when creating a block. This makes a block creation resource-intensive and deters hackers from modifying the contents of a block. The linkage between the previous and current blocks through hash values provides added security against 
data tampering in the blockchain. A hash value of a block also summarises the contents of the block header, working as an identifier for the block.

\subsubsection{Consensus mechanism}

Different blockchains use different mechanisms to create blocks, known as the consensus mechanism. Popular consensus mechanisms include Proof of Work, Proof of Stake, and Delegated Proof of Stake. In Proof of Work, miners need to perform certain mathematical work to be able to create a block (Dinh et al., 2018). In bitcoin, miners need to find a hash value smaller than the target value using SHA-256. ${ }^{4}$ For example, to increase the difficulty, the bitcoin software can increase the number of zeros required at the beginning of the hash value and make the target value smaller. Therefore, miners have to perform multiple trial-and-error operations to find a nonce that generates a hash value lower than the target value. Based on the protocol, the target value is regularly adjusted to allow a block generation every ten minutes on average (Nakamoto, 2008; Yli-Huumo et al., 2016; Zheng et al., 2017 Dinh et al., 2018). Once a miner solves the problem, he/she can create a block with the calculated hash value. Proof of Work, however, is computationally demanding and consumes a lot of electricity, enough to power a small country like Ireland for a year (Hern, 2017). It also creates inefficiency and resource waste as other unsuccessful miners' work becomes futile.

Proof of Stake eases a computational demand depending on the stake of block-creating nodes, reducing energy consumption and resource waste. As in Proof of Work, block-creating nodes still need to find a hash value smaller than the target value. However, in Proof of Stake, the difficulty of task reduces as a block-creating node puts a higher stake in the network. ${ }^{5}$

In Delegated Proof of Stake ${ }^{6}$, full nodes can put their stakes to vote for a delegate, and the delegate with the highest votes creates a block (Zheng et al., 2017 Smith, 2018). Because delegates are chosen from other nodes, they have incentives to provide valuable service to the blockchain to attract more votes. See Dinh et al. (2018) for other consensus mechanisms.

\footnotetext{
${ }^{4}$ Proof of Work requires miners to find a hash value lower than the target value. Hence: $H(n \| H(b))<t$

where $\mathrm{H}$ is a hash function, $\mathrm{b}$ is the content of the block, $\mathrm{n}$ is a nonce, and $\mathrm{t}$ is a target threshold.

${ }^{5}$ Proof of Stake reduces the difficulty of the task for a high-stake block-creating node by increasing the target value. Specifically:

where $\mathrm{s}$ is the stake function of a miner, $\mathrm{M}$.

$$
H(n \| H(b))<s(M) \cdot t
$$

6 Using algorithm, this technology-based democracy feature in blockchain enhances decentralisation and discourages centralisation.
} 


\subsection{Ledger update}

\subsubsection{Block validation}

Once a block is created, it is broadcast to other full nodes. As blockchain operates in a trustless environment between nodes, each full node validates the block before adding it to its own copy of the blockchain (Fernandez-Carames and Fraga-Lamas, 2018; Saberi et al., 2019). If the block is invalid (e.g., the block contains invalid transactions or has a hash value greater than the difficulty target), other nodes will ignore the block. Even if a few nodes accept the invalid block and add it to their copy of the blockchain, the majority will ignore it and not use it for the subsequent block development. Therefore, the copies of the few nodes which accepted the invalid block will soon become outdated, discouraging the acceptance of invalid blocks. On the other hand, if the block is valid, it will be accepted by other nodes and will be used for the subsequent block development. As new blocks are appended to the block, it becomes more secure in the blockchain (Dinh et al., 2018). Even if some nodes do not accept the valid block and do not add it to their copies of the blockchain, the majority will accept the block and continue developing subsequent blocks after that. Therefore, nodes which rejected the valid block will soon have an outdated copy of the blockchain, discouraging the rejection of a block without valid reasons. In another case, it is also possible to receive multiple valid blocks at the same time. This occurs when different block-creating nodes find valid (but different) blocks at the same time. In that case, for example, some nodes will receive Block A as a valid block while others receive Block $B$ as a valid block depending on their proximities to the blockcreating nodes. These are called 'forks' - the existence of multiple chains in the blockchain (Zheng et al., 2017). The forks caused by the conflicting blocks exist temporarily in the blockchain. The protocol of the blockchain determines which chain becomes the active (or main) chain. For example, according to the longest chain rule, the chain that has more subsequent blocks becomes the active chain (Nakamoto, 2008). Therefore, in our example, as subsequent blocks are created and added to Block A or B, only one chain (let us say, Block A's chain) becomes the active chain. Nodes that received Block B, on the other hand, reorganise their chain to be consistent with the active chain. The transactions included in Block B but not included in Block A are sent back to the Mempool for the inclusion in the subsequent block (Morrow, 2014). Therefore, blockchain operates in a system that leads to a consensus even though each node keeps its own copy of the blockchain and does not necessarily trust each other. 


\subsubsection{Transaction confirmation}

Once a block is validated and added to the blockchain, the transactions included in the block become confirmed. Specifically, their status changes from 'unconfirmed' to 'confirmed'. A confirmed block initially receives a value of 1 . However, owing to the possibility of forks, it is recommended for users to wait for further confirmations to ensure that their transactions are irreversible. Each time a new block is appended to the block, the number of confirmations increases for the transaction. In bitcoin, transactions are considered irreversible once confirmations reach number six (Costea, 2019; Nakamoto, 2008). Our transaction, where Alice wants to pay Bob, is now confirmed and recorded in the blockchain in an irreversible manner.

\section{Blockchain and taxation}

Research into blockchain and various aspects of taxation has developed rapidly over the past five or six years. The development of IT and AI is often referred to as the fourth industrial revolution (Vishnevsky and Chekina, 2018), as mentioned earlier (see also Hong and Seo, 2018), and has considerable relevance for tax. The on-going research links tax with IT (see Beck et al., 2017), although Risius and Spohrer (2017), reviewing research published up to 2017, also identify computer systems, law, finance and political science as subject areas displaying research work, with 'users and society', 'intermediaries', 'platforms' and 'firms and industries' being the levels of analysis. They identify taxation as a possible area of future study in relation to 'firms and industries' (Risius and Spohrer, 2017, p. 402). The interest in the topic is worldwide, with the USA and Eastern Europe being particularly well represented in the literature (see, for the latter Guzov et al., 2018; Ulyanava, 2018; Emelianova and Dementyev, 2020). Much of the available literature is still only available as conference presentations/papers, revealing its topicality. Nearly all the papers one reads commence with an explanation of how blockchain works, often accompanied by details about taxation, to make the areas accessible to a wider audience, and many concentrate on how the writer's/writers' own jurisdiction deals with blockchain (e.g., Warnez (2017) on Denmark; van Rijswijk et al. (2018) on The Netherlands). Some papers are far more technologically based than others (see, for example, Ainsworth and Alwohaibi, 2017a, 2017b; Ainsworth et al., 2017; Pokrovskaia, 2017; Ainsworth, Alwohaibi and Cheetham, 2020, Ainsworth, Alwohaibi, Cheetham and Tirand, 2020). There seem to be three main themes within this growing body of academic 
literature: (i) the type of assets or income (new tax bases) made possible by the technology (e.g., the various cryptocurrencies, of which bitcoin is perhaps the best known) and how to tax them; (ii) how the technology itself might affect individual taxes and tax collection/administration systems; and (iii) whether the technology will increase or decrease the potential for tax evasion and/or avoidance. ${ }^{7}$ These themes are considered below.

\subsection{The type of assets or income and how to tax them}

There is considerable difficulty and debate about how to classify and deal with the assets/income that blockchain makes possible, notably the various forms of cryptocurrencies (see Nellen, 2015; Fulmer, 2018). Ahmed (2018, p. 703) notes that as at January 2018, there were 1,384 cryptocurrencies, although Ulyanava $(2018$, p. 1) gives a figure of "more than 2,000", with bitcoin, ethereum, bitcoin cash, ripple, and litecoin being the common ones. As a cryptocurrency can be much more than the digital equivalent of cash (although many do not aim to be anything more than this), then various taxation issues arise, not least because the cryptocurrencies are themselves developing and changing much faster than the legal systems which need to deal with them (see Akins et al., 2014; Schwanke, 2017). These systems are not consistent, even within one country. Ahmed (2018, p. 705) cites the USA as an example, saying that " $[\mathrm{t}]$ he extraordinary diversity in the treatment of cryptocurrency is troubling" and gives details of cases that have already reached the courts where debate about classification and treatment have been key elements. Generally, the trend is to liken a given form of cryptocurrency to something that is already covered by existing tax code (see Ahmed, 2018), although some jurisdictions have specifically set out how they will treat cryptocurrency transactions. ${ }^{8}$ As well as a direct equivalent of cash, cryptocurrencies might be considered as types of property (USA); assets (Israel); income (Slovenia); a medium for barter transactions (Singapore); a financial instrument (Germany); an investment contract; a commodity; or specific tokens carrying the right to goods/services on top of any monetary value, which may also be associated with a particular place or project. Cryptocurrencies may additionally possess hybrid features. ${ }^{9}$ Ahmed $(2018$, p. 712) illustrates this by reference to the Legends cryptocurrency (LGD):

\footnotetext{
${ }^{7}$ Demirhan (2019) covers these areas in some detail.

${ }^{8}$ Ulyanava (2018) gives a useful summary of how cryptocurrencies are classified in a number of different jurisdictions. See also Ahmed (2018, pp. 713-715) and Liedel (2018) for a discussion of various countries' different classifications.

${ }^{9}$ They can be split to fulfil separate functions (see Webb, 2018; Lerer, 2019).
} 
LGD is redeemable for drinks and dances at a strip club in Las Vegas and can give special VIP privileges to those individuals who possess a large amount of it. Functionally, it works more as property since its circulation is limited to use at this club - it does not have the breadth of use as normal cryptocurrency that might be widely accepted by many merchants though it may still be redeemable for cash on major exchanges.

The tax treatment is inherently far from straightforward, and potentially different between different types of cryptocurrency, and between countries. The various properties that might be possessed by a cryptocurrency if it is a hybrid instrument could potentially result in differing tax treatments of the same item. Even if considered as a straight cash substitute by a user, a tax classification may still cause problems. As Ahmed (2018, p. 713) comments:

If taxed as property, then users must realize a tax gain or loss every time they transact in the cryptocurrency. This requirement becomes quite burdensome on daily users of cryptocurrency. If someone buys a burger at a fast food restaurant, they will be required to declare a gain or loss on the intrinsic movement in the value of the cryptocurrency while possessing it. This task is complicated by the fact that these cryptocurrencies can fluctuate in price tremendously within a single day.

Breier et al. (2019) explore in more detail the possibilities attached to tokens, in a Swiss context, considering three particular types conferring rights: (i) the right to a share of a company's future profits (income-sharing tokens); (ii) the right to a share of future royalties generated by means of a specified technology (royalty-sharing tokens); and (iii) the use of technology to be developed by the issuing entity (utility tokens). They detail the possible legal classifications of these items and how they would be potentially taxed under Swiss tax law, noting that they are "new investment tools [that] are difficult to assess based on the existing tax law framework and the existing tax practice" (Breier et al., 2019, p. 1168): treatments of similar items elsewhere might be completely different. HM Customs \& Revenue (HMRC) in the UK have considered in detail issues relating to bitcoin, setting out how various transactions would be dealt with by existing UK taxes, but have said that "[t]he tax treatment of security tokens and utility tokens will be addressed in future guidance" (HM Customs \& Revenue, 2019).

The issue of cryptocurrencies in the first place (often referred to as 'mining') may also give rise to tax complications - again depending on the rights conferred. Akins et al. (2014, p. 
41) comment that there are two possible ways to tax (specifically) bitcoin issue: "(1) as an accession of wealth upon receipt of a bitcoin reward, or (2) as compensation received for services rendered within the bitcoin system. Each treatment differs significantly in the character and timing of income tax realization". The first sees the bitcoin 'miner' as creating an intangible asset, new value for him-/herself, and the second as compensation for the 'miner' for the services rendered in creating the asset. Again, this is jurisdiction-specific and Akins et al. (2014) write in a US context. Ahmed (2018) considers 'initial coin offerings' (ICOs) that raise money through crowdfunding and give purchasers a right to royalties in a project. He comments on the many tax questions surrounding such ICOs, and asks (2014, p. 704):

Should ICOs be treated as pure income? ICOs are used as an alternative to raising funding through conventional venture funding, so should there be some element of equity treatment? Typically, the party or parties that initiate an ICO will hold a portion of the coins and then keep the funds from selling all of the other coins. Should there be a requirement to mark-to-market? These currencies can fluctuate significantly from one week to the next (sometimes $1,000 \%$ or more).

These questions, along with those about the treatment of cryptocurrencies after they have been issued, are ones which the world's different tax systems are struggling to answer.

\subsection{How blockchain might affect individual taxes and tax collection/administration systems}

A considerable amount of work has been done on Value Added Tax (VAT) and the implications of blockchain and/or similar technologies by Richard Ainsworth and colleagues. Ainsworth and Shact (2016) report a survey of experts, suggesting that we might expect to see tax collected by governments via blockchain by 2023, on average, although some thought this would not happen before 2025. The governments were not specified, nor the type of tax, although Ainsworth and Shact (2016, p. 2) suggest that the EU will be an early adopter for VAT, as it "will bring substantial efficiencies to VAT collection. It will reduce costs and build critical inter-governmental trust relationships". Blockchain will, they comment, be crucial in implementing the EU Commission's April 2016 Action Plan on VAT. This and subsequent papers (e.g., Ainsworth and Alwohaibi, 2017a, 2017b; Ainsworth et al., 2017; Ainsworth, Alwohaibi and Cheetham, 2020, Ainsworth, Alwohaibi, Cheetham and Tirand, 2020) discuss various countries' proposals to implement a blockchain system to deal with VAT, explaining in depth and with detailed diagrams how the different proposals would work, and the necessary 
electronic infrastructure required to implement them. They favour the proposal put forward by the Gulf Cooperation Council (GCC) as addressing many of the flaws perceived in the EU system, as it was designed 'from scratch', rather than being superimposed on an existing system. Nemade et al. (2019) map out diagrammatically how a blockchain taxation system would work and cite VAT or Goods Sales Tax (GST) as areas of potential application - but note that the complexity of a tax system and lack of IT specialists militate against implementation. Ainsworth and Viitasaari (2017) look at payroll and conclude that this is an area ripe for the use of blockchain technology, but is complex, and would benefit from trials in smaller applications, such as by small companies. Leiding et al. (2016) apply the use of ethereum to dealing with vehicle taxation, among other vehicle-related issues, while Wijaya et al. (2019) look at the potential for the use of blockchain for stamp duty in an Indonesian context.

Owens (2017, p. 7) considers that blockchain technology has the potential to modernise tax administration systems, but requires "considerable networking effects" before it can be implemented, although in the longer term "blockchain can significantly improve tax compliance by guaranteeing real-time, automated tax payments from the tax payer to the state budget at the time when a transaction in [sic] being executed". This would ensure the benefits of compliance in real time, reduction in transaction costs and elimination of the risks of fraud and tax evasion. It would also have the potential (per Owens, 2017, p. 8) to address multinational enterprises' transfer pricing problems, as the inherent lack of information about comparable transactions between related parties could be eliminated and could be extended for use by Customs and Excise bodies. However, Owens (2017, p. 8) concludes that for all this to work, "a supporting eco-system and a 'whole of government' approach is needed", with widespread use of blockchain and appropriate regulatory measures - implicit also in a recent UK Office of Tax Simplification (OTS) report, considering technology (OTS, 2019).

Considerable concern remains about how the use of the technology will affect data audit requirement and privacy (Bossa and de Paiva Gomes, 2019), and whether other data management technology (e.g., Oracle, SQL) which have been more widely tested, might be more appropriate. 'Permissioned' access might address some of the perceived problems (Hoffman, 2018). Another aspect of blockchain not often considered is its own intensive use of resources (although this has been mentioned earlier in this paper). Truby (2018, p. 399) studies "existing regulatory and fiscal policy approaches towards digital currencies in order to provide a basis for further legal and policy tools targeted at mitigating energy consumption of 
Blockchain technologies", as well as considering the potential of blockchain for use in mitigating climate change.

\subsection{Whether blockchain will increase or decrease potential for tax evasion and/or avoidance}

The work by Ainsworth and colleagues, as referred to above, considers especially the potential inherent in blockchain to eliminate VAT fraud and evasion, saying that "[m]ost importantly, blockchain will immediately end revenue losses well in excess of $€ 50$ to $€ 60$ billion per year in missing trader intra-community fraud (MTIC)" (Ainsworth and Shact 2016, p. 2). Ainsworth et al. (2017), Ainsworth, Alwohaibi and Cheetham (2020), and Ainsworth, Alwohaibi, Cheetham and Tirand (2020) suggest that the introduction of a specific tax crypto-tax-currency, which they term 'VATCoin', would allow governments to track VAT payments that are due, and which it has not been hitherto possible to do. To track VAT frauds, governments have usually to follow the supply trail by looking at invoices. In conjunction with the use of digital/fiscal invoices, the use of VATCoin would significantly reduce the opportunity for VAT frauds, although the authors acknowledge that VATCoin is open to some vulnerabilities. Wijaya et al. (2017) also think that blockchain can reduce the incidence of fraud in the area of VAT.

Emeliana and Dementeyev (2020) suggest that blockchain technology can reduce tax evasion, but the fact that most states are implementing legal systems to address it on an individual basis, and not in any coordinated way, may provide (unintended) opportunities for 'tax arbitrage' (whereby the tax rules of one state are 'traded off' against the rules of another, to gain tax advantage). Akins et al. (2014) and Marian (2019) also noted the potential for tax evasion, owing to the anonymity inherent in the anonymous nature of digital currencies. A further problem arises in that blockchain technology itself does not take account of international borders. As Marian (2019, p. 554) comments, disintermediation (or decentralisation) means that no one party controls, operates or maintains the technology, and once a blockchain operation is active, "national borders and regulations and regulations within them become largely irrelevant". It can only be changed with the majority of the users agreeing - and they may be scattered worldwide. Moreover, resiliency and 'temper resistance' mean that a transaction cannot be changed - so a fraud cannot be undone. Marian (2019) shows how these features, combined with the anonymity of users (or pseudonymity), and a deliberate choice not to regulate blockchain activities, have allowed many former tax havens to re- 
establish themselves as 'cryptocurrency havens', offering secrecy, thus negating much of the "[r]ecent coordinated activities against tax havens [that] significantly hampered tax havens ability to trade in secrecy" (Marian, 2019, p. 555), and opening up new means of tax evasion and avoidance, which will require an internationally coordinated response to defeat, possibly involving regulation of blockchain applications prior to release (Marian, 2019, p. 566).

Other authors are less clear about whether the use blockchain will deter or facilitate tax evasion (e.g., Houben and Snyers, 2018). Hegadekatti and Yatish (2016), for instance, suggest that tax evasion may be facilitated in the short term, but that the technology makes it easier to detect tax evaders in the longer term. Faccia and Mosteanu (2019a) suggest that the checks and balances inherent within blockchain, combined with a relatively low tax rate, would help eliminate evasion, as would allowing access to transactions to tax authorities, in the case of companies (Yew, 2018).

In summary, the literature on tax and blockchain reveals considerable inconsistency between different jurisdictions (and sometimes within the same jurisdiction) about the classification and treatment of cryptocurrencies, exacerbated by the inherent complexity of tax law and the speed at which technological developments outstrip the ability of legal systems to keep pace with those developments. While governments are clearly interested in the potential blockchain offers for tax systems themselves, developing this potential is hampered by the lack of IT specialists and a fear that technology will create further opportunities for tax evasion and avoidance, not only in terms of 'tax arbitrage', but in the creation of cryptocurrency havens. Providing solutions would, arguably, require international coordination in and cooperation in the development of law on a level hitherto unimaginable.

\section{Blockchain and the banking industry and capital markets}

The compendium of literature on big data analytics and blockchain technology has ignited a new debate about the implications of blockchain technologies for businesses and transaction processing systems (Peters and Panayi, 2016; Hassani et al. 2018; Eduardo, 2020). Estimations from the World Economic Forum (2015), suggest that 10\% of the world's GDP will be stored on blockchains or blockchain related technology by 2025. Blockchain offers a major breakthrough in data storage, simultaneity in transaction information processing and improved transaction security (Tandulwadikar, 2016; Miyamae et al., 2018; Wang et al., 2020). Additionally, previous studies show a significant interlink between blockchain cryptocurrency 
and other multidisciplinary sectors (such as banking, energy instruments, commodity assets, debt and equity market, international trade financing, smart contracting, etc. (Huynh, Burggraf and Wang, 2020, Huynh, Shahbaz, Nasir and Ullah, 2020). For example, blockchain technology has fundamentally transformed the existing banking and financial operating models into a new round of financial technological (fintech) innovation. Studies show that, blockchain is influencing disruptions in the banking industries as well as reshaping the technological innovation in the financial sector (Tandulwadikar, 2016; Cong and He, 2019). There is irrefutable evidence that cryptocurrency is the typical application in blockchain world. That said, there are several misgivings associated with blockchain technology. For example, there are concerns such as spillover risk on cryptocurrency markets as a result of high market volatility of cryptocurrencies based price (Huynh et al., 2018), bad news associated with cybersecurity attacks of the network (Huynh et al., 2018, Huynh, 2019), etc. Further, there is a high possibility of contagion risk that will cause serious damage to other key financial sectors in the event of cryptocurrencies crashing (Huynh et al., 2018). Although blockchain is run by computers and considered as a transparent process, human factors, such as individual sentiments, personal biases, etc., can impair its efficient operations (Nasir et al., 2019, Burggraf et al., 2020).

This study contributes to the prior blockchain and data analytics literature in three ways: (i) it provides new insights and perspectives concerning the effects of blockchain on banking transactions, debt and equity finance administration, smart contracts, capital market transparency, etc.; (ii) the findings from this study will provide a unique and useful insight that will motivate academics and future researchers to examine other implications of blockchain technologies for banking and finance industry; and (iii) the study will offer a better understanding to professionals, managers and decision makers regarding the practical applications of blockchain technology in transaction processing.

\subsection{Blockchain technologies lessen transaction costs}

From the perspective of the banking industry, blockchain has contributed to reducing transaction and processing costs (Tandulwadikar, 2016; Miyamae et al., 2018; Witzig and Salomon, 2018). Blockchain technology has strengthened the pursuit of transparency, accountability and trust within the complex banking and finance ecosystem (Nguyen, 2016; Miyamae et al., 2018; Eduardo, 2020). Blockchain technology will have far-reaching economic 
and social implications, which are not yet foreseeable to their full extent. It will reduce transaction costs by disintermediating many well established sectors and most incumbent businesses from the banking and finance sectors and provide opportunities for newcomers (Witzig and Salomon, 2018). For example, combinations of blockchain technology and big data analytics have created opportunities for different fintech companies to establish banking services with lower start-up costs (Iskander, 2017).

Traditional banking ledger accounts (like any other business ledger accounts) serve as the main information source for the preparation of financial statements and consolidated reporting. Most banks process significant amount of transactions daily using less secure and less transparent transaction processing systems (Balakrishnan and Ertan, 2018; Balsmeier and Vanhaverbeke, 2018; Faccia and Mosteanu, 2019b). Also, if these banks were to update their ledgers each time a transaction occurred, the ledger accounts would quickly become cluttered and errors may be inevitable (Miyamae et al., 2018; Faccia and Mosteanu, 2019b;). A typical commercial banking accounting ledger includes a master file containing all customers' current account balances together with previous transactions for a specified period, summary ledgers for different operations of the banks that keep track of cash and asset movements, and a transaction processing system. Blockchain offers opportunities for streamlining the complex traditional banking processes as well as ensuring secure and transparent transactions (Tandulwadikar, 2016; Iskander, 2017; Hassani et al., 2018).

\subsection{Blockchain provides transparency in debt and equity capital administration}

There was major concern regarding banks' debt and equity records after the 2007/08 credit crisis. Banks' debt and equity financing continues to be a subject of suspicion due to the massive high level corporate scandals and the 2007/08 financial crisis that led to the failures of well-known and large high street banks (Balakrishnan and Ertan, 2018). Banks usually categorise their capital transactions under three main sub-headings. These include bank loans and collateral, bond issuance and stock issues. Bank loans are mainly reported in the form of notes payable accounts, mainly used to provide medium- or long-term financing and typically asset-backed as collateral. These financial instruments entail complex financial transaction processes that can be dealt with more effectively by blockchain technology. For example, the notes payable ledgers are used to record outstanding notes, including information such as maturity dates, interest rate, opening balance and closing balance. Blockchain technology 
provides a more secure and transparent database on the notes payable ledger to enhance smarter decisions. The bonds payable ledger summarises bond issuance transactions, which includes information about contractual obligations such as interest rate payments, accumulated interest, issuer information, maturity periods, etc. Stock issuances are recorded in the equity capital ledger which notes amounts of stock issued, classification of stock issued, total accumulated stock balance, etc. Blockchain technology ensures transparency as well as streamlining the debt and equity transactions of banks (Iskander, 2017; Hassani et al., 2018; Eduardo, 2020). The application of blockchain hashing and consensus to banking transaction and banks' ledger creation will enhance efficient accounting records, data integrity, data security and provide a more reliable audit trail for bank transactions.

\subsection{Blockchain enhances bank international transfer and makes export credits faster}

Blockchain technology can introduce significant improvement into banking activities especially in the context of international trade financing, such as issuing a letter of credit and smart commercial contracts (Peters and Panayi, 2016; Tapscott and Tapscott, 2017; Ikeda and Hamid, 2018; Cong and He, 2019). Previously, most banks have suffered complications and bottlenecks in executing transactions involving international financing. Issuing a letter of credit requires a lot of communication and exchange of complex financial documents among multiple participants around the world. These participants include importers, issuing banks, nominated banks, advising banks, negotiating banks, confirming banks, insurance companies and even port authorities. Please refer to Figure 2 below. 


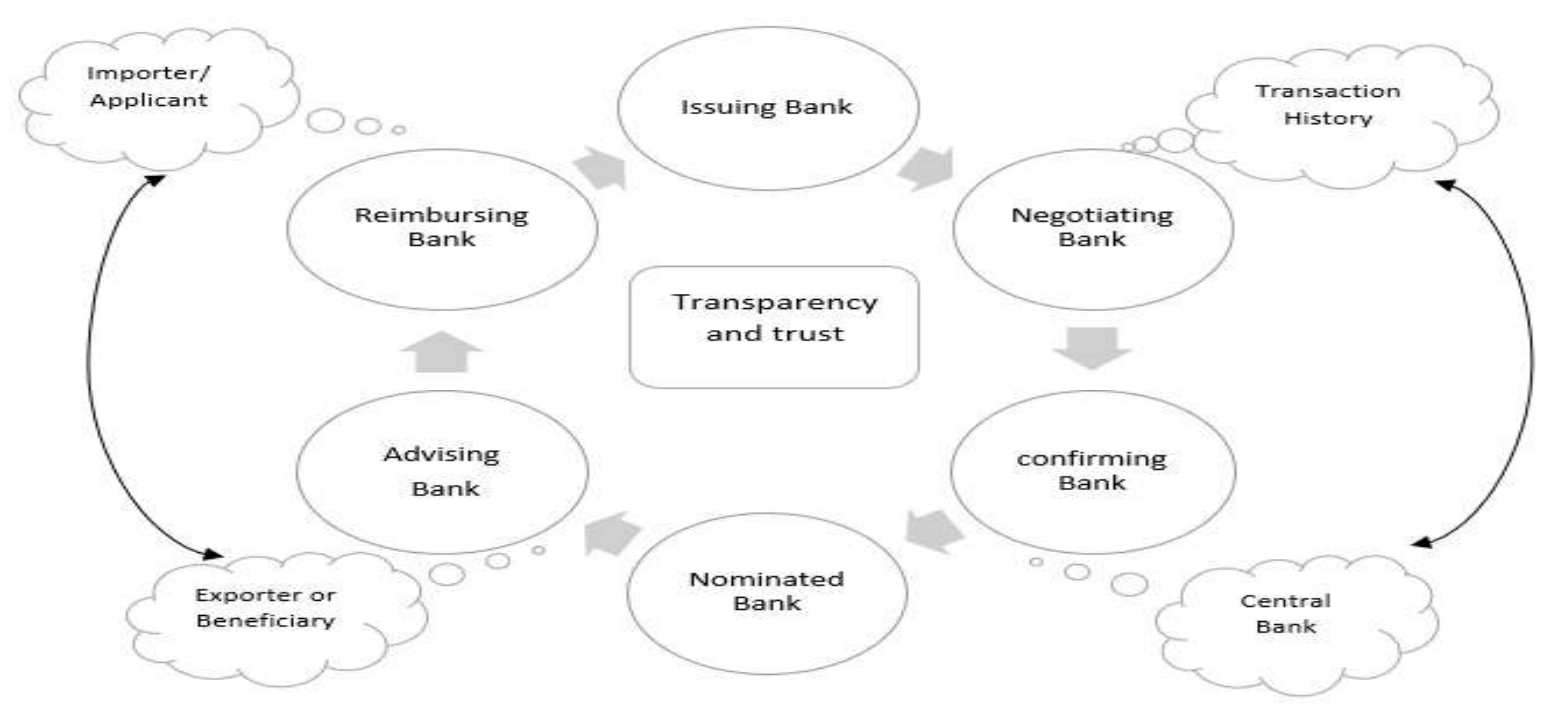

Fig. 2. Blockchain enhances smart contracting, big data analytics, ${ }^{10}$ transparency and trust among banks in foreign transactions, from Ahmed (2020).

Banks have to go through several operational formalities and protocols in executing foreign transactions. For example, Figure 2 above illustrates some of the operational protocols and formalities that banks go through in executing international transactions and payments. Blockchain technology provides a smart database that enhances transparency and can be accessed by all the parties involved in Figure 2 above for better and smarter decisions. The banks involved first and foremost can use the database generated by the blockchain technology to check the transaction history of the beneficiaries against an internal and external sanction list to ascertain their creditworthiness and integrity. Participating banks can also use the blockchain database to ensure that central banks and others comply with regulations. The financing bank may require syndicated loan arrangers (agents) to enable banks involved to agree on long-term security guarantees against funds' disbursement and credit repayments. On the other hand, the advising banks will provide advice (based on smart information collected from the blockchain database) to the exporter about the cost and benefits associated with the letter of credit and communicate with the issuing bank and the confirming bank for acceptance or amendments before approving the request.

\footnotetext{
10 The World Economic Forum (2015) estimated that about 10\% of GDP will be stored on blockchains or blockchain-related technology by 2025 . It is expected that the blockchain digital ledger will provide throughput via a valuable, relevant, reliable, inimitable, etc., database that will enhance innovation, business intelligence and decision analytics among the banking and finance ecosystems. Blockchain is expected to revolutionise the entire banking and finance sector (Iskander, 2017).
} 
The issuing bank must secure payment maturity as well and ensure that treasury has adequate funds and availability of Nostro accounts (accounts held in foreign currency) before sending transfer instructions to the bank holding Nostro accounts. Also, when payments are made, all the parties involved in the transactions must be notified again. Blockchain provides technology that decentralises all the above transactions and operations for the parties involved in the above transactions other than the banks. Blockchain promotes smart contracts, and distributed ledger accounts to enable all parties to monitor the activities in the chain. Blockchain provides consensus by using hashing technology as well as streamlining all processes, reducing bottlenecks, reducing errors associated with the above transactions, speeding up the issuance of the letter of credit, and also minimising disputes. In addition, an exporter will have faster access to credit with added transparency and trust guaranteed by blockchain. The distributed ledger enables the banks involved to share intelligence as well as the tax and transaction history of customers (Iskander, 2017; Wang et al., 2019; Eduardo, 2020). With the appropriate privacy considerations, the model can be further extended to create globally verifiable blockchain identities for all participants for sharing the sanction list as well as enhancing real time transactions at a reduced cost (Hassani et al., 2018; Ikeda and Hamid, 2018; Cong and He, 2019).

\section{Blockchain and corporate governance}

Corporate governance includes a set of firm level and country level monitoring mechanisms aiming to enhance the accountability and transparency of corporate entities. Internal corporate governance mechanisms include: (a) board of directors; (b) managerial incentives; (c) capital/ownership structures; (d) by-laws and charter provisions; and (e) internal control systems. On the other hand, external corporate governance mechanisms include jurisdictionalspecific laws and regulations, capital markets (including debt and stock markets), credit rating agencies, and other external regulatory mechanisms (Gillan, 2006). It is argued that traditional corporate governance mechanisms (transactions) are likely to be facilitated as well as disrupted by blockchain technology. National and transnational regulatory bodies are gradually developing policies and procedures to bring such innovative products/companies into their regulatory architecture. For example, the Twitter hashtag (\#GoingDigital) was widely used in the Organisation for Economic Cooperation and Development (OECD) blockchain event in 2018. Major capital markets around the world are now considering the possibility of applying blockchain technology in recording the stocks issued by companies and in capturing the 
subsequent trading activities of such stocks (Yermack, 2017). The 'digital wallet' within the distributed ledger technology might be helpful in tracing the identity of shareholders. For investors, it is less costly to trade shares as the use of blockchain minimises the role of clearing houses in processing share-based transactions. More recently, in 2019, the London Stock Exchange has allowed the issuance of shares using 'blockchain tokens' and the financial technology company, '2030', was a pioneering company in raising $£ 3$ million equity, which was also regulated by the Financial Conduct Authority Sandbox 4 programme which aims to regulate highly innovative products and services in the UK (The Telegraph, 2019). Discussing the wider implications of blockchains for other types of assets, Yermack (2017, p. 8) argues that "entrepreneurs are actively investigating blockchains' suitability for recording ownership of a wide range of assets, from stocks and bonds to real estate, automobile titles, luxury handbags, and works of art". The speed, cost, and data integrity associated with these financial innovations and distributed ledger systems will certainly enhance the 'timely record keeping' problem relating to who owns their shares in real time (i.e., individual investors, institutional investors, corporations, charities, governments, families and other blockholders).

Blockchain technology will also enormously improve shareholder engagement systems. Lack of shareholder engagement is a corporate governance issue highlighted in the corporate governance codes of most countries around the world. Recent research predicts that blockchain will significantly affect the dynamics of powers between shareholders, managers, and the board of directors (Yermack, 2017). Issues relating to employees' stock ownership, such as insider trading and backdating of stock options, would be nearly impossible, as participants in some types of blockchain will not be able to change the original entries. This sophisticated double entry ledger system will therefore enhance transparency in all share-based transactions. Every single user in the distributed ledger system (network) can observe the trading pattern of managers, corporate raiders and activists, and this is another feature relating to the disclosure and transparency of blockchain technology. Shareholder voting could only with difficulty be manipulated in appointing board members in the annual general meeting. It will be much easier for a shareholder to have a 'digital vote/e-vote' in a blockchain network. Digital voting can reduce the costs associated with voting, holding annual general meetings, and will certainly enhance voting turnout (Lafarre and Van der Elst, 2018).

Blockchain will also reshape the corporate reporting and disclosure landscape in the coming decades. Corporate information will be easily and freely accessible, and companies will be able to submit returns to various parties and regulatory bodies in real time. According to the Financial Reporting Council (2018), from a preparer perspective, information reported 
by companies is more likely to be: (a) cost effective; (b) easy; (c) compatible; and (d) timely. Similarly, from a user's perspective, information reported using blockchain will be: (a) engaging; (b) credible; (c) useable; and (d) contextual. Corporate information (strategic report, financial reports, key performance indicators, etc.) can be disclosed in a flexible way, by applying a variety of disclosure media (text, videos, etc.). Such disclosure blocks can be verified and countersigned by the auditors, which can eventually be electronically filed in the national storage system (NSM), as demonstrated in Figure 3 below.

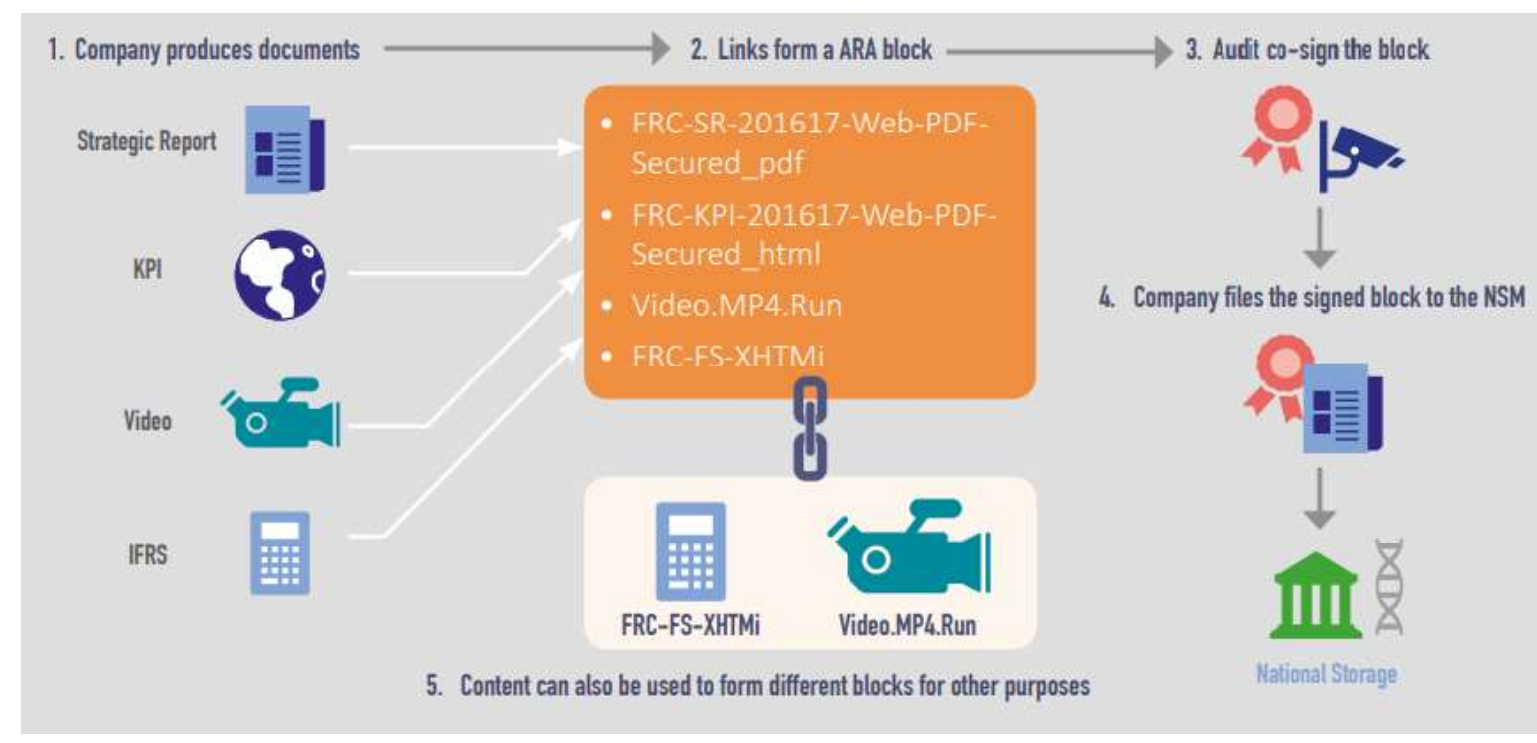

Fig. 3. Blockchain and the boundary of reporting, from Financial Reporting Council (2018). The box highlighted orange in the center indicates that various reporting documents such as video, pdf, web-based content can all be collated instantly in one block.

In summary, blockchain technology is expected to facilitate corporate governance, corporate ownership, shareholders' voting, and communication with shareholders (through enhanced financial reporting). The introduction of blockchains in global capital markets will also eliminate issues relating to stock manipulation, insider trading, and backdating of stock options. In addition to that, transparency and accountability is an important feature of corporate governance and blockchain technology will further enhance the accountability and transparency issues in listed companies. 


\section{Blockchain and international trade}

Given the recent 'hype' surrounding blockchain technology (Berg et al., 2019; Smith and Dhillon, 2019), it is important to understand how blockchain adds value to international trade both at the micro- and macro-levels. At the micro-level, the globalisation of value chains and the onerous demands to meet diverse regulatory requirements and regimes increases the complexities of international production networks. In addition, the multi-echelon nature and the geographically disjointed entities which operate in culturally diverse regions (Brady et al., 2001) show the promise of a distributed ledger system in ensuring transparency, traceability and security of international business (Saberi et al., 2019). Blockchain technology's core strength lies in its ability publicly to validate, record and distribute transactions in immutable and encrypted data which facilitate global sourcing, production and distribution networks. Moreover, the creation of decentralised currencies and self-executing digital contracts (smart contracts) and intelligent assets that can be controlled online (smart property) seem to explain the efficiency-seeking, resource-seeking and asset-seeking nature of multinational corporations (Dunning and Lundan 2008; Franco et al., 2008). As international trade is primarily influenced by the internalisation demands of firms as well as the localisation determinants of nations (see Buckley and Casson, 2019), blockchain technology could enhance a secured exchange of firmspecific resources in a distributed manner. Also, corporations would not have to change their structure and governance systems or reconfigure relationships to reflect institutional demands in different countries. In this regard, Wang et al. (2019) note that blockchain creates permanent shareable and actionable records of a product/service's digital footprint throughout the entire supply system if they are integrated with field-sensing technologies such as the internet of things. Among the key perceived benefits of blockchain in international trade is its ability to overcome the three main challenges facing global trade, consisting of institutional differences (North, 1991), psychic distance (O'Grady and Lane, 1996) and the opportunistic behaviours (Lumineau and Verbeke, 2016) of global value chains. Whilst these three main factors have been explored in a wide range of international trade literature, there has not been much discussion that identifies disruptive technology as a possible solution.

First, for several years, institutional differences have been identified as a source of cost to international trade due to the void which usually exists between international firm's level of knowledge (meso-level) and its new environment (macro-level). Hence, blockchain technology has the potential to enable firms to overcome institutional differences and costs, which has been the subject of much debate over the years (see North, 1991; Aguilera and Grøgaard, 2019; Golesorkhi et al., 2019). Institutions matter in international trade because its topographical 
landscapes create trade barriers and operational costs. This means that multinational companies (MNCs) operating in different environments face challenges in strategically locating their value chains and adapting their operations to the diversity of institutions across countries and regions (Jackson and Deeg, 2008). Operational costs for MNCs trading within economies of the Global South (thus, less developed, transitional and emerging), with different systems of political governance and social structures, continuously highlight the importance of institutions in understanding business strategy and corporate financial performance across national borders. In the Global North, for example, the reformulation of the Lisbon Treaty to include the efficient Functioning of the European Union (TFEU) has been introduced to deal with institutional costs of international trade among EU countries. A critical assessment of Article 3(3) of the TFEU, shows that the EU is still grappling with finding solutions to redirect competition laws and regimes to strengthen market forces whilst simultaneously protecting social actors and free movement of goods and services (see Venit, 1996; Šmejkal, 2015). Blockchain technology seems to present the capability to curb the oligopolistic actions and reactions of large corporations, production cartels, anti-competitive agreements and abuses of market power through mergers and acquisitions between Global North and South countries. Legal and political institutions around the world could apply country-specific as well as the World Trade Organization's (WTO) rules and regulations for corporate financial transactions (see, for instance, World Trade Organization, 2015a, 2015b), using the track and analyse function of blockchain technology in monitoring activities of global trade (Bonsón and Bednárová, 2019; Moll and Yigitbasioglu, 2019).

Secondly, blockchain has also been the subject of much academic debate with no clear answers in the international trade literature. It has been argued in the literature that firms trade abroad by moving into countries that are psychically close before venturing into more distant countries (O'Grady and Lane, 1996). The central problem of the psychic distance theory is whether closeness to a market enhances a firm's knowledge of the contextual realities prevailing in that particular country or whether the gradual sequence followed by firms to acquire progressively the knowledge needed to trade in other countries could be shortened by the application of relevant technology, such as the internet of things, artificial intelligence and blockchain technology. What is not explicit in the conventional literature that explains the factors influencing global trade is what firms need to do to minimise the uncertainties as well as the amount of time needed to enter into different countries to trade. Similarly, Kogut and Singh (1988) and Hult et al. (2020) conclude that psychically close countries are easier for companies to learn from. This seems to present the assumption that psychically close countries 
are more similar and that such similarities promote international trade more than dissimilarity. Despite the obvious weaknesses posed by trade barriers due to psychic distances, the distributed architecture that underpins the revolutionary, yet disruptive, blockchain technology seems to offer the opportunity to change cultures of transparency and trust within cyberspaces. This means that psychic distances could be eradicated by using democratic and accountable cyberspaces to facilitate trade between nations. WTO trade agreements, which highlight core principles of liberalisation and permitted exceptions involving an individual country's commitments to lowering customs, tariffs and removing trade barriers, could be aligned with the neo-liberal opportunities offered by crypto-technologies to enhance free and fair systems of trade between nations. From commodity markets to governance systems, blockchain could support the removal of hindrances that for many years have affected developing and transitional economies. For most of the $20^{\text {th }}$ and $21^{\text {st }}$ centuries, least developed, developing transitional, frontier, and even some emerging, economies continue to struggle to obtain fair prices for commodities as they grapple with the consequences of colonialism amidst issues of sustainable systems of co-production and consumption (pro-sumption) of goods and services.

Thirdly, the principal-agent theory and transaction cost analysis have been subjects of tremendous debate in the international trade literature (Contractor, 2007; Chang et al., 2019; Moll and Yigitbasioglu, 2019). The primary focus of the issues presented by the principalagent paradox is the degree of opportunism that exists within transnational activities as boundedly rational managers make decisions aimed at maintaining their hegemony through complex governance structures in overseas business transactions. Opportunism in general and opportunistic behaviours, according to Saberi et al. (2019), are critical constructs within the transaction cost economics and agency literature as firms could, for instance, make suppliers highly dependent through the creation of artificial shortages, establishment of cartels and the use of transfer pricing (see Sikka, 2010). Opportunistic managers could satisfice their selfinterest by organising international trading transactions through complex global value chains in a modular, relational, hierarchical, captive and market-based approaches to take undue advantage over market actors (Saberi et al., 2019). In such circumstances, AI, the internet of things and, most crucially, cryptographic algorithms could be used to cypher information to provide substantial insights into the near-shoring, offshoring, near-sourcing and outsourcing systems of global value chains. In addition, blockchain technology could broaden perspectives on sustainability, environmental uncertainties and identify artificial barriers created by special interest firms (within monopolistic and oligopolistic sectors) that block fair access into 
international markets. One way, therefore, to ensure a stable and sustainable international trade is equal access to immutable market information.

At the macro-level, blockchain technology has the potential to influence trade finance, improve customs' procedures and provide timely information about the origin of goods and services. McDaniel and Norberg (2019) indicate that using a distributed ledger reduces the expense and time required to facilitate trade between business to business (B2B), government to government $(\mathrm{G} 2 \mathrm{G})$ and other stakeholders that rely on third-party lending or insurance. Ganne (2018) and Chang et al. (2019) argue that customs' procedures could be expedited to boost trade volumes at a lower cost, improve the detection of illicit trade flows and deter illegitimate efforts to circumvent international trade rules. Whilst some authors offer critical perspectives on a centrally regulating body for blockchain technology to guarantee its versatility (Herian, 2018), the opportunities presented by disruptively decentralising business interactions means that law enforcement agencies could explore the benefits whilst mitigating the potential risks posed by the technology in international trade. Consequently, since the Financial Times article written by Arnold in 2018 stressing that blockchain can no longer be ignored, there has been growing attention paid to this phenomenon.

Measures to facilitate free and fast movement of goods and services between nations have gained enormous attention from policy makers around the world. Yet, apart from the technicalities and efficiencies espoused by the few who are interested in speculative bitcoin investments, there is no evident attempt to assess empirically the implications of blockchain technology for the broader aspects of the global economy. For instance, whilst the trading policies of the WTO and other regional trading blocs, such as the EU, Mercosur, Pacific Alliance and NAFTA, focus on strengthening border procedures to ensure an effective leveraging of the absolute and comparative economic advantage of nations, technology such as the internet of things, AI and blockchain has not been well explored as a means to facilitate trade between nations at the conceptual, policy and practical level. Blockchain technology, therefore, presents an opportunity to facilitate trading procedures by supporting the digitisation of cross-border movement of goods and services (Ganne, 2018). Choi (2012) pinpoints that institutional differences, including burdensome customs' procedures and multiple government agencies responsible for consumer protection, create duplicative administrative procedures which tend to increase costs for Global South countries. These antecedents explain the growing importance of trade facilitation technologies and measures that enhance secured import and export transit processes. Ganne (2018) asserts that several economies have followed the United Nations Conference on Trade and Development's (UNCTAD) proposal to automate customs 
systems, such as the Automated Systems for Customs Data (ASYCUDA) to boost integrated platforms in standardising international trade procedures. Cryptographic letters of credit (CLC) could serve as proof of trust among trading partners with secured payments for cross-border exchange of goods and services. On the one hand, B2B, such as importers, exporters and financial institutions, could use CLC to share immutable data and documents between local and international stakeholders for trade facilitation. B2G and G2G interactions, on the other hand, could use blockchain to enhance inter-agency cooperation and the protection of crossborder intellectual properties, copyrights and patents. Blockchain technologies could expose the protectionist agenda of different nations (Norberg, 2019) and improve the transparency of cross-border trade to support decision making concerning foreign direct investments (McDaniel and Norberg, 2019). Moreover, it would lead to an accurate measurement of production, pricing and distribution targets of certain commodities, such as crude oil, which has always been susceptible to volatility, sometimes arising from political rumours within OPEC and other oil-producing nations (see Andoni et al., 2019; Lu et al., 2019).

\section{Summary and conclusion, and avenues for future research}

This paper sought to conceptualise the implications of blockchain technology for business operations, especially in the accounting and finance industry, with a particular focus on how it is revolutionising taxation, banking, capital markets, governance structures and international trade. The paper demonstrates some significant benefits of the technology concerning how organisations could utilise it to reduce operational, transaction and agency costs. The unique technical capabilities of blockchain could change organisational and national cultures and help enhance transparency and trust within cyberspaces. Combined with fieldsensing technologies, AI and the internet of things, blockchain has the potential to offer significant benefits for international trade, capital markets, taxation, auditing, banking, and corporate governance, among other areas. Its revolutionary yet disruptive potential has been touted as presenting global opportunities to shift socio-economic and political conventions. Some studies have been critical of the technology by arguing that the unregulated, solutionistdriven nature of blockchain, to some extent, seems to exist within the boundaries of anarchocapitalists who use it as a source of financial and political power with scant regard for political, legal and democratic accountability within the collective interest of other stakeholders. Consequently, blockchain technology may be perceived as creating a neo-modernised form of capitalism that makes corporations difficult to govern, as it may further the creation of 
plutocratic societies whereby capital is managed and controlled by the ruling, elite and wealthy class of individuals. Moreover, given the current disconnection between the low profits of the productive economy and the high profits of the finance economy, the scalability of blockchain technology raises more questions than answers regarding sustainability, 'fairness' and 'equitable' application of the law to ordinary citizens and owners of capital at the local and global levels. Hence, several regulatory agencies are not well prepared to support the growing use of the technology. In terms of blockchain and tax systems, it can be summarised that there are several issues relating to the complexity of tax law and legal systems that may not necessarily keep pace with these recent technological developments. While governments around the world are clearly interested in adapting potential blockchain for tax systems themselves, developing this potential is hampered by the lack of IT specialists. A review of recent corporate governance literature also suggests that blockchain will enhance: (a) financial reporting transparency; (b) shareholders' ownership and voting system; and (c) the stock market trading mechanisms.

Despite all the implications discussed above, the introduction of blockchain technology has also implications for business schools, and the higher educational institutions around the world. Blockchain technology related teaching and learning content will play a major role in reshaping the traditional curriculum in many business schools. Many universities in the UK have already started undergraduate and postgraduate degrees in financial technology in collaboration with computer sciences and IT departments. Such interdisciplinary programmes will not only be able to equip students with necessary technical skills but will also encourage academics in carrying out multidisciplinary research.

This paper concludes that since blockchain technology can no longer be ignored, further empirical studies are required to evaluate the regulatory implications of scalability. Moreover, the primary issues of performance, hidden potential and the long-term value to global supply chains need to be empirically examined and their shortcomings addressed. In addition, whilst solutions to the limitations of blockchain technology have been conceptually examined, several of these studies fail to provide concrete empirical assessment, and so further studies are needed to focus on the wider governance implications of rolling out the technology more extensively. Future studies can explore the perception of auditors (Big Four accounting firms) to understand the degree of acceptability and resistance to various types of financial technologies, including blockchain and cryptocurrencies. Similarly, the adoption of blockchain technology will also affect the quality of financial reporting, and hence it will be interesting to explore how the adoption of this technology will affect the quality of financial reporting in different 
jurisdictions. A comparative analysis of the financial reporting quality of firms adopting blockchain with non-adopters could provide meaningful insights about the quality of information produced using the distributed ledger technology (blockchain).

\section{References}

Abadi, J. and Brunnermeier, M., 2018. Blockchain Economics, National Bureau of Economic Research, working paper No. 25407, doi: 10.3386/w25407.

Agrawal, H., 2019. What is the bitcoin Mempool \& why it matters??, from https://coinsutra.com/bitcoin-mempool/, accessed 10 May 2020.

Aguilera, R. V. and Grøgaard, B., 2019. The dubious role of institutions in international business: A road forward, Journal of International Business Studies, 50(1), 20-35.

Ahluwalia, S., Mahto, R. V. and Guerrero, M., 2020. Blockchain technology and startup financing: A transaction cost economics perspective, Technological Forecasting and Social Change, 151(February), 119854, doi: 10.1016/j.techfore.2019.119854.

Ahmed, K., 2020. YouTube podcast on blockchain and the banking industry, from https://youtu.be/rq3SW-KUKhg, accessed 7 May 2020.

Ahmed, S., 2018. Cryptocurrency \& robots: How to tax and pay tax on them, South Carolina Law Review, 69(3), 697-740.

Ainsworth, R. T. and Alwohaibi, M., 2017a. Blockchain, bitcoin, and VAT in the GCC: The missing trader example, Boston University School of Law, Law and Economics Research Paper No. 17-05, from SSRN https://ssrn.com/abstract=2919056 or doi: 10.2139/ssrn.2919056, accessed 1 May 2020.

Ainsworth, R. T. and Alwohaibi, M., 2017b. The first real-time blockchain VAT - GCC solves MTIC fraud (July 24, 2017), Boston University School of Law, Law and Economics Research Paper No. 17-23, from SSRN https://ssrn.com/abstract=3007753 or doi: 10.2139/ssrn.3007753, accessed 3 May 2020.

Ainsworth, R. T., Alwohaibi, M., Cheetham, M. and Tirand, C., 2017. A VATCoin proposal following on the 2017 EU VAT proposals - MTIC, VATCoin, and BLOCKCHAIN, Boston University, School of Law, Law and Economics Research Paper No. 18-09, available at SSRN: https://ssrn.com/abstract=3151465, accessed 4 May 2020.

Ainsworth, R. T., Alwohaibi, M. and Cheetham, M., 2020. UK \& KSA VATs: A cutting-edge proposal - mini-blockchain and VATCoin, from SSRN: https://ssrn.com/abstract=3574381 ordoi: 10.2139/ssrn.3574381, accessed 3 May 2020.

Ainsworth, R. T., Alwohaibi, M., Cheetham, M. and Tirand, C., 2020. A VATCoin proposal following on the 2017 EU VAT proposals - MTIC, VATCoin, and BLOCKCHAIN, Boston University School of Law, Law and Economics Research Paper No. 18-09, from SSRN: https://ssrn.com/abstract=3151465, accessed 6 May 2020.

Ainsworth, R. T. and Shact, A., 2016. Blockchain (distributed ledger technology) solves VAT fraud, Boston University School of Law, Law and Economics Research Paper No. 1641, from SSRN https://ssrn.com/abstract=2853428 or doi: 10.2139/ssrn.2853428, accessed 1 May 2020. 
Ainsworth, R. T. and Viitasaari, V., 2017. Payroll tax \& the blockchain, Tax Notes International, 13 March, 1007-1024, Boston University, School of Law, Law and Economics Research Paper No. 17-17, from SSRN https://ssrn.com/abstract=2970699, accessed 1 May 2020.

Akins; B. W., Chapman, J. L. and Gordon, J. M., 2014. A whole new world: Income tax considerations of the bitcoin economy, Pittsburgh Tax Review, 12(1), 25-56.

Andoni, M., Robu, V., Flynn, D., Abram, S., Geach, D., Jenkins, D. and Peacock, A., 2019. Blockchain technology in the energy sector: A systematic review of challenges and opportunities, Renewable and Sustainable Energy Reviews, 100, 143-174.

Angelis, J. and Ribeiro da Silva, E., 2019. Blockchain adoption: A value driver perspective, Business Horizons,62(3), 307-314. doi: 10.1016/j.bushor.2018.12.001.

Appelbaum, D. and Smith, S. S., 2018. Blockchain basics and hands-on guidance: Taking the next step toward Implementation and Adoption, CPA Journal, 88(6), 28-37.

Arnold. M., 2018. Davos: Blockchain can no longer be ignored, Financial Times, 24 January, from https://www.ft.com/content/c0794556-ff50-11e7-9650-9c0ad2d7c5b5, accessed 5 May 2020.

Asolo, B., 2018. Full node and lightweight node, from https://www.mycryptopedia.com/fullnode-lightweight-node/, accessed 10 May 2020.

Asolo, B., 2019. Blockchain public key \& private key: A detailed guide, from https://www.mycryptopedia.com/public-key-private-key-explained/, accessed 10 May 2020.

Balakrishnan, K. and Ertan, A., 2018. Banks' financial reporting frequency and asset quality, Accounting Review, 93(3), 1-24.

Balsmeier, B. and Vanhaverbeke, S., 2018. International financial reporting standards and private firms' access to bank loans, European Accounting Review, 27(1), 75-104.

Beck, R., Avital, M., Rossi, M. and Thatcher, J. B., 2017. Blockchain technology in business and information systems research, Business \& Information Systems Engineering, 59(6), $381-384$.

Beedham, M., 2019. All you need to know about Bitcoin network nodes, from https://thenextweb.com/hardfork/2019/03/01/bitcoin-blockchain-nodes-network/, accessed 10 May 2020.

Berg, C., Davidson, S. and Potts, J., 2018. Some public economics of blockchain technology (2 March 2018), from SSRN https://ssrn.com/abstract=3132857 or http://dx.doi.org/10.2139/ssrn.3132857, accessed 20 February 2020.

Berg, C., Davidson, S. and Potts, J., 2019. Capitalism after Satoshi: Blockchains, dehierarchicalisation, innovation policy, and the regulatory state, Journal of Entrepreneurship and Public Policy, 9(2), 152-164, doi: 10.1108/JEPP-03-2019-0012.

Bitcoin Project, 2020. Running A Full Node. retrieved from https://bitcoin.org/en/fullnode\#what-is-a-full-node, accessed 10 May 2020.

Bonsón, E. and Bednárová, M., 2019. Blockchain and its implications for accounting and auditing, Meditari Accountancy Research, 27(5), 725-740, doi: 10.1108/MEDAR-112018-0406. 
Bossa, G. and de Paiva Gomes, E., 2019. Blockchain: Technology as a tool for tax information exchange or an instrument threatening the taxpayer's privacy?, from SSRN https://ssrn.com/abstract $=3540277$ or doi: $10.2139 /$ ssrn.3540277, accessed 4 May 2020.

Brady, M. K., Robertson, C. J. and Cronin, J. J., 2001. Managing behavioral intentions in diverse cultural environments: An investigation of service quality, service value, and satisfaction for American and Ecuadorian fast-food customers. Journal of International Management, 7(2), 129-149.

Breier, M., Hongler, P. and Winzap, M., 2019. Taxing tokens - a Swiss perspective on the taxation of initial coin offerings and blockchain-based tokens, Tax Notes International, 89(12), 1159-1168.

Brennan, N. M., Subramaniam, N. and van Staden, C. J., 2019. Corporate governance implications of disruptive technology: An overview, British Accounting Review, 51(6), 100806, doi: 10.1016/j.bar.2019.100860.

Brophy, R., 2019. Blockchain and insurance: A review for operations and regulation, Journal of Financial Regulation and Compliance, 28(2), 215-234, doi: 10.1108/JFRC-09-20180127.

Buckley, P. and Casson, M., 2019. Decision-making in international business. Journal of International Business Studies, 50(8), 1424-1439.

Burggraf, T., Huynh, T. L. D., Rudolf, M. and Wang, M., 2020. Do FEARS drive Bitcoin?, Review of Behavioral Finance, doi: 10.1108/RBF-11-2019-0161.

Cai, C. W., 2018. Disruption of financial intermediation by FinTech: A review on crowdfunding and blockchain, Accounting and Finance, 58(4), 965-992. doi: 10.1111/acfi.12405.

Cawrey, D., 2014. What are bitcoin nodes and why do we need them?, from https://www.coindesk.com/bitcoin-nodes-need, accessed 10 May 2020.

Chang, S. E., Chen, Y. C. and Wu, T. C., 2019. Exploring blockchain technology in international trade. Industrial Management \& Data Systems, 119(8), 1712-1733.

Choi, Y. B., 2012. Institutions for economic prosperity - an entrepreneurial perspective, in Choi, Y. B. (ed), Institutional Economics and National Competitiveness (pp. 63-83). New York, Routledge.

Cong, L. W. and He, Z., 2019. Blockchain disruption and smart contracts. Review of Financial Studies 32(5), 1754-1797.

Contractor, F. J., 2007. Is international business good for companies? The evolutionary or multi-stage theory of internationalization vs. the transaction cost perspective. Management International Review, 47(3), 453-475.

Costea, V., 2019. Do bitcoin transactions need six confirmations?, from https://bitcoinmagazine.com/articles/mathematics-research-suggests-sixconfirmations-arent-really-needed-bitcoin-transactions, accessed 10 May 2020.

Coyne, J. G. and McMickle, P. L., 2017. Can blockchains serve an accounting purpose?, Journal of Emerging Technologies in Accounting, 14(2), 101-111, doi: 10.2308/jeta51910. 
Curran, B., 2020. What is a Merkle Tree? Beginner's guide to this blockchain component, from https://blockonomi.com/merkle-tree/, accessed 10 May 2020.

Dai, J. and Vasarhelyi, M. A., 2017. Toward blockchain-based accounting and assurance, Journal of Information Systems, 31(3), 5-21, doi: 10.2308/isys-51804.

Davidson, S., De Filippi, P. and Potts, J., 2016. Economics of Blockchain (8 March 2016), from SSRN https://ssrn.com/abstract=2744751 or doi: org/10.2139/ssrn.2744751, accessed 20 February 2020.

Demirhan, H., 2019. Effective taxation system by blockchain technology, in Hacioglu, U. (ed), Blockchain Economics and Financial Market Innovation (pp. 347-360), The Netherlands, Springer.

di Castri, S., Grasser, M. and Kulenkampff, A., 2018. Financial authorities in the era of data abundance: Regtech for regulators and suptech solutions, from SSRN https://ssrn.com/abstract=3249283 or doi: org/10.2139/ssrn.3249283, accessed 19 February 2020.

Digital Signatures, 2012. Retrieved from https://docs.microsoft.com/en-us/previousversions/windows/it-pro/windows-2000-server/cc962021(v=technet.10), accessed 10 May 2020.

Dinh, T. T. A., Liu, R., Zhang, M., Chen, G., Ooi, B. C. and Wang, J., 2018. Untangling blockchain: A datapProcessing view of blockchain systems, IEEE Transactions on Knowledge and Data Engineering, 30(7), 1366-1385, doi: 10.1109/TKDE.2017.2781227.

Dunning, J. H. and Lundan, S. M., 2008. Multinational Enterprises and the Global Economy, $2^{\text {nd }}$ edition, Cheltenham, Edward Elgar Publishing.

Eduardo Demarco, A., 2020. Analysing blockchain/distributed ledger technology in capital markets and know your customer process. Journal of Securities Operations \& Custody 12(1), 58-71.

Emelianova, N. N. and Dementyev, A. A., 2020. Cryptocurrency, taxation and international law: Contemporary aspects, artificial intelligence: Anthropogenic nature vs. social origin, in ISC Conference - Volgograd, in Popkova, E. and Sergi, B. (eds.) Advances in Intelligent Systems and Computing, Volume 1100 (pp. 725-731), Cham, Springer.

EU Commission, 2016. Communication from the Commission to the European Parliament, the Council and the European Economic and Social Committee, on an action plan on VAT: Towards a single EU VAT area - time to decide, COM(2016) 148 Final (7 April), available at https://eur-lex.europa.eu/legalcontent/EN/TXT/?uri=COM\%3A2016\%3A148\%3AFIN, accessed 10 May 2020.

European Union, 2012. Treaty of Lisbon Amending the Treaty on European Union and the Treaty Establishing the European Community, 26 October 2012, 2012/C326/17, from https://eur-

lex.europa.eu/LexUriServ/LexUriServ.do?uri=CELEX:12012E/TXT:EN:PDF, accessed 21 May 2020.

Evans, J., n.d. Blockchain nodes: How they work (all types explained), from https://nodes.com/, accessed 10 May 2020.

Faccia, A. and Mosteanu, N. R., 2019a. Tax evasion information system and blockchain, Journal of Information Systems \& Operations Management, Summer, 65-74. 
Faccia, A. and Mosteanu N. R., 2019b. Accounting and blockchain technology: from doubleentry to triple-entry, Business \& Management Review 10(2), 108-116.

Fernandez-Carames, T. M. and Fraga-Lamas, P., 2018. A review on the use of blockchain for the Internet of Things, IEEE Access, 6, 32979-33001, doi: 10.1109/ACCESS.2018.2842685.

Financial Reporting Council, 2018. Blockchain and the future of corporate reporting, from https://www.frc.org.uk/getattachment/58866565-ab3b-44d3-93e11ef7158968d5/Blockchain-and-the-future-of-corporate-reporting-how-does-itmeasure-up-(June-2018).pdf, accessed 27 April 2020.

Franco, C., Rentocchini, F. and Vittucci Marzetti, G., 2008. Why do firms invest abroad? An analysis of the motives underlying foreign direct investments (15 December 2008), from SSRN https://ssrn.com/abstract=1283573 or doi: $10.2139 /$ ssrn. 1283573 , accessed 17 April 2020.

Frankenfield, J., 2018. Master node, from https://www.investopedia.com/terms $/ \mathrm{m} / \mathrm{master}-$ node-cryptocurrency.asp, accessed 10 May 2020

Frankenfield, J., 2019a. Block time, from https://www.investopedia.com/terms/b/block-timecryptocurrency.asp, accessed 10 May 2020.

Frankenfield, J., 2019b. Nonce, from https://www.investopedia.com/terms/n/nonce.asp, accessed 10 May 2020.

Fulmer, N., 2018. Exploring the legal issues of blockchain applications, Akron Law Review, 52(1), 161-192.

Ganne, E., 2018. Can blockchain revolutionize international trade?, Geneva, World Trade Organization. doi: 10.30875/7c7e7202-en.

Gillan, S. L., 2006. Recent developments in corporate governance: An overview. Journal of Corporate Finance, 12(3), 381-402.

Goffard, P.O., 2019. Fraud risk assessment within blockchain transactions, Advances in Applied Probability, 51(2), 443--467.

Golesorkhi, S., Mersland, R., Randøy, T. and Shenkar, O., 2019. The performance impact of informal and formal institutional differences in cross-border alliances, International Business Review, 28(1), 104-118.

Gomber, P., Kauffman, R. J., Parker, C. and Weber, B. W., 2018. On the fintech revolution: Interpreting the forces of innovation, disruption, and transformation in financial services, Journal of Management Information Systems, 35(1), 220-265. doi: 10.1080/07421222.2018.1440766.

Gomber, P., Koch, J. and Siering, M., 2017. Digital finance and fintech: Current research and future research directions, Journal of Business Economics, 87(5), 537-580.

Gonzalez, L., 2019. Blockchain, herding and trust in peer-to-peer lending, Managerial Finance. doi: 10.1108/MF-09-2018-0423.

Guzov, I., Soboleva, D. and Artemova D., 2018. Digital technologies in accounting and taxation: Some issues from Russian literature and experience, paper presented at the 3rd International Economic Symposium (IES).

Harris, W. L. and Wonglimpiyarat, J., 2019. Blockchain platform and future bank competition, Foresight, 21(6), 625-639. doi: 10.1108/FS-12-2018-0113. 
Hassani, H., Huang, X. and Silva, E., 2018. Banking with blockchain-ed big data. Journal of Management Analytics, 5(4), 256-275.

Hegadekatti, K. and Yatish, S. G., 2016. Examining taxation of fiat money and bitcoins vis-avis regulated cryptocurrencies, from SSRN https://ssrn.com/abstract=2846645 or doi: 10.2139/ssrn.2846645, accessed 4 May 2020.

Herian, R., 2018. Regulating blockchain: Critical Perspectives in Law and Technology, Abingdon, Routledge.

Hern, A., 2017. Bitcoin mining consumes more electricity a year than Ireland, from https://www.theguardian.com/technology/2017/nov/27/bitcoin-mining-consumeselectricity-ireland, accessed 10 May 2020.

HM Customs \& Revenue, 2019. Policy paper, Cryptoassets: tax for businesses, updated 20 December 2019, from https://www.gov.uk/government/publications/tax-oncryptoassets/cryptoassets-tax-for-businesses, accessed 24 July 2020.

Hoffman, M., 2018. Can blockchains and linked data advance taxation?, paper presented at the WWW Conference, Track, 3rd Workshop on Linked Data \& Distributed Ledgers, 2327 April, Lyon, France.

Hong, S. and Seo, C. R., 2018. Developing a blockchain information based accounting and tax information in the 4th industrial revolution, Journal of the Korea Convergence Society, $9(3), 45-51$.

Houben, R. and Snyers, A., 2018. Cryptocurrencies and blockchain: Legal context and implications for financial crime, money laundering and tax evasion, European Parliament, Policy Department for Economic, Scientific and Quality of Life Policies, available at repository.uantwerpen.be, accessed 1 May 2020.

Hult, G. T. M., Gonzalez-Perez, M. A. and Lagerström, K., 2020. The theoretical evolution and use of the Uppsala Model of internationalization in the international business ecosystem. Journal of International Business Studies, 51(1), 38-49.

Huynh, T. L. D., 2019. Spillover risks on cryptocurrency markets: A look from VAR-SVAR Granger causality and student's t-copulas, Journal of Risk and Financial Management 12(2), 52 .

Huynh, T. L. D., Burrgraf, T. and Wang, M., 2020. Gold, platinum, and expected bitcoin returns, Journal of Multinational Financial Management, 100628. doi:10.1016/j.mulfin.2020.100628.

Huynh, T. L. D., Nguyen, S. P., and Duong, D., 2018. Contagion risk measured by return among cryptocurrencies, in Anh, L., Dong, L., Kreinovich, V. and Thach, N. (eds), Econometrics for Financial Applications, ECONVN 2018, Studies in Computational Intelligence, Volume 760 (pp. 987-998), Cham, Springer.

Huynh, T. L. D., Shabaz, M, Nasir, M. A. and Ullah, S., 2020. Financial modelling, risk management of energy instruments and the role of cryptocurrencies, Annals of Operations Research. doi: 10.1007/s10479-020-03680-y.

Iansiti, M. and Lakhani, K. R., 2017 The truth about blockchain, Harvard Business Review, 33(2), 360-381. doi: 10.1016/j.annals.2005.11.001.

Ikeda, K. and Hamid M. N., 2018. Applications of blockchain in the financial sector and a peerto-peer global barter web, in Raj, P. and Deka, G. C. (eds), Advances in Computers, 
Volume 111, Blockchain Technology: Platforms, Tools and Use Cases (pp. 99-120), The Netherlands, Elsevier.

Iskander, M., 2017. Blockchain: The future of all data, Intellectual Property and Technology Law Journal, 22(1), 1-18.

Islam, A. K. M. N., Mäntymäki, M. and Turunen, M., 2019. Why do blockchains split? An actor-network perspective on bitcoin splits, Technological Forecasting and Social Change, 148, 119743. doi: 10.1016/j.techfore.2019.119743.

Jackson, G. and Deeg, R., 2008. Comparing capitalisms: Understanding institutional diversity and its implications for international business, Journal of International Business Studies, 39(4), 540-561.

Jamison, M. A. and Tariq, P., 2018. Five things regulators should know about blockchain (and three myths to forget), Electricity Journal, 31(9), 20-23. doi: 10.1016/j.tej.2018.10.003.

Johnson, D., Menezes, A. and Vanstone, S., 2001. The Elliptic Curve Digital Signature Algorithm (ECDSA), International Journal of Information Security, 1(1), 36-63. doi: $10.1007 / \mathrm{s} 102070100002$.

Karajovic, M., Kim, H. M. and Laskowski, M., 2019. Thinking outside the block: Projected phases of blockchain integration in the accounting industry, Australian Accounting Review, 29(2), 319-330. doi: 10.1111/auar.12280.

Kogut, B. and Singh, H., 1988. The effect of national culture on the choice of entry mode. Journal of International Business Studies, 19(3), 411-432.

Kokina, J., Mancha, R. and Pachamanova, D., 2017. Blockchain: Emergent industry adoption and implications for accounting, Journal of Emerging Technologies in Accounting, 14(2), 91-100. doi: 10.2308/jeta-51911.

Kuznetsov, A., Pushkar'ov, A., Kiyan, N. and Kuznetsova, T., 2018, May. Code-based electronic digital signature, in 2018 IEEE 9th International Conference on Dependable Systems, Services and Technologies (DESSERT (pp. 331-336), Kiev, IEEE.

Lafarre, A. and Van der Elst, C., 2018. Blockchain technology for corporate governance and shareholder activism (March 2018), European Corporate Governance Institute (ECGI) - Law Working Paper No. 390/2018, Tilburg Law School Research Paper No. 2018-7, from SSRN https://ssrn.com/abstract=3135209 or doi: 10.2139/ssrn.3135209 (accessed 10 May 2020).

Lai, R. and Chuen, D. L. K., 2018. Blockchain-From Public to Private, in Chuen, D. L. K. and Deng, R. (eds), Handbook of Blockchain, Digital Finance, and Inclusion, Volume 2, ChinaTech, Mobile Security, and Distributed Ledger (pp. 145-177), The Netherlands, Elsevier.

Learn about Ethereum, 2020. from https://ethereum.org/learn/, accessed 10 May 2020.

Lee, I. and Shin, Y. J., 2018 Fintech: Ecosystem, business models, investment decisions, and challenges, Business Horizons, 61(1), 35-46. doi: 10.1016/j.bushor.2017.09.003.

Lee, J. Y., 2019. A decentralized token economy: How blockchain and cryptocurrency can revolutionize business, Business Horizons, 62(6), 773-784. doi: 10.1016/j.bushor.2019.08.003.

Li, X., Jiang, P., Chen, T., Luo, X. and Wen, Q., 2020. A survey on the security of blockchain systems, Future Generation Computer Systems, 107, 841-853. 
Liedel, D. A., 2018. The taxation of bitcoin: How the IRA views cryptocurrencies, Drake Law Review, 66(1), 107-146.

Leiding, B., Memarmoshrefi, P. and Hofgrefe, D., 2016. Self-managed and blockchain-based vehicular ad-hoc networks, in UbiComp '16: Proceedings of the 2016 ACM International Joint Conference on Pervasive and Ubiquitous Computing: Adjunct, September (pp. 137-140), from doiorg.ezproxy.nottingham.ac.uk/10.1145/2968219.2971409, accessed 4 May 2020.

Leong, C., Tan, B., Xiao, X., Tan, F. T. C. and Sun, Y., 2017. Nurturing a fintech ecosystem: The case of a youth microloan startup in China, International Journal of Information Management, 37(2), 92-97. doi: 10.1016/j.ijinfomgt.2016.11.006.

Lerer, M., 2019. The taxation of cryptocurrency: Virtual transactions bring real-life tax implications, CPA Journal, 89(1), 40-43.

Lewis, A., 2018. The Basics of Bitcoins and Blockchains: An Introduction to Cryptocurrencies and the Technology that Powers Them, Florida, Mango Publishing Inc.

Lu, H., Huang, K., Azimi, M. and Guo, L., 2019. Blockchain technology in the oil and gas industry: A review of applications, opportunities, challenges, and risks, IEEE Access, 7, 41426-41444.

Lumineau, F. and Verbeke, A., 2016. Let's give opportunism the proper back seat, Academy of Management Review, 41(4), 739-741.

Marian, O., 2019. Blockchain havens and the need for their internationally-coordinated regulation, North Carolina Journal of Law \& Technology, 20(4), 529-568.

Marshall, B., 2018. How does ECDSA work in bitcoin, from https://medium.com/@blairlmarshall/how-does-ecdsa-work-in-bitcoin-7819d201a3ec, accessed 10 May 2020.

McDaniel, C. A. and Norberg, H. C., 2019. Can blockchain technology facilitate international trade?, (24 April 2019), Mercatus Research Paper, from SSRN https://ssrn.com/abstract $=3377708$ or doi: $10.2139 /$ ssrn.3377708, accessed 21 April 2020.

Miyamae, T., Honda, T., Tamura, M. and Kawaba, M., 2018. Performance improvement of the consortium blockchain for financial business applications, Journal of Digital Banking 2(4), 369-378.

Moll, J. and Yigitbasioglu, O., 2019. The role of internet-related technologies in shaping the work of accountants: New directions for accounting research, British Accounting Review. Elsevier Ltd, 51(6), 100833. doi: 10.1016/j.bar.2019.04.002.

Moos, M., 2019. Bitcoin active nodes outnumber XRP active addresses, from https://cryptoslate.com/bitcoin-active-nodes-outnumber-xrp-active-addresses/, accessed 10 May 2020.

Morkunas, V. J., Paschen, J. and Boon, E., 2019. How blockchain technologies impact your business model, Business Horizons, 62(3), 295-306. doi: 10.1016/j.bushor.2019.01.009, accessed 19 February 2020.

Morrow, J., 2014. What is an orphan block?, from https://blog.cex.io/bitcoin-dictionary/whatis-an-orphan-block-9632, accessed 10 May 2020. 
Nakamoto, S., 2008. Bitcoin: A peer-to-peer electronic cash system, from https://bitcoin.org/bitcoin.pdf, accessed 19 February 2020.

Nakashima, T., 2018. Creating credit by making use of mobility with FinTech and IoT, IATSS Research, 42(2), 61-66. doi: 10.1016/j.iatssr.2018.06.001.

Nasir, M. A., Huynh, T. L. D., Nguyen, S. P. and Duong, D., 2019 Forecasting cryptocurrency returns and volume using search engines, Financial Innovation 5(1), Article 2. doi: 10.1186/s40854-018-0119-8.

Nellen, A., 2015. Taxation and today's digital economy, Journal of Tax Practice \& Procedure, April-May, 27-37.

Nemade, A.E., Kadam, S.S., Choudhary, R.N., Fegade, S.S. and Agrawal, K., 2019. Blockchain technology used in taxation, International Conference on Vision Towards Emerging Trends in Communication and Networking (ViTECoN), Vellore, India, pp. $1-4$.

Nguyen, Q. K., 2016. Blockchain - a financial technology for future sustainable development. 2016 3rd International conference on green technology and sustainable development (GTSD), Kaohsiung, 2016, pp. 51-54, doi: 10.1109/GTSD.2016.22.

Nomura Research Institute, 2016. Survey on blockchain technologies and related services, from https://www.meti.go.jp/english/press/2016/pdf/0531_01f.pdf, accessed 3 May 2020 .

Norberg, H. C., 2019. Unblocking the bottlenecks and making the global supply chain transparent: How blockchain technology can update global trade (13 March 2019), The School of Public Policy Publications, Volume 12:9 March 2019, from SSRN https://ssrn.com/abstract=3353818, accessed 28 April 2020.

North, D. C., 1991. Institutions, Journal of Economic Perspectives, 5(1), 97-112.

Nowiński, W. and Kozma, M., 2017. How can blockchain technology disrupt the existing business models?, Entrepreneurial Business and Economics Review, 5(3), 173-188. doi: 10.15678/EBER.2017.050309.

Office of Tax Simplification (OTS), 2019. Technology review: A vision for tax simplicity, available at https://www.gov.uk/government/publications/will-tax-simplification-stillbe-needed-as-technology-advances, accessed 30 January 2019.

O'Grady, S. and Lane, H. W., 1996. The psychic distance paradox, Journal of International Business Studies, 27(2), 309-333.

Owens, J., 2017. Blockchain 101 for governments, Wilton Park Conference, 27-29 November, WP1566, from wiltonpark.org, accessed 1 May 2020.

Penas, O., Plateaux, R., Patalano, S. and Hammadi, M., 2017. Multi-scale approach from mechatronic to Cyber-Physical Systems for the design of manufacturing systems, Computers in Industry, 86, 52-69. doi: 10.1016/j.compind.2016.12.001.

Peters, G. W. and Panayi, E., 2016. Understanding modern banking ledgers through blockchain technologies: Future of transaction processing and smart contracts on the internet of money. Banking beyond banks and money, in Tasca, P., Aste, T., Pelizzon, L., and Perony, N. (eds) Banking Beyond Banks and Money. New Economic Windows (pp. 239-278), Cham, Springer. 
Phan, L., Li, S. and Mentzer, K., 2019. Blockchain technology and the current discussion on fraud, Computer Information Systems Journal Articles, https://digitalcommons.bryant.edu/cisjou/28, accessed 25 July 2020.

Pokrovskaia, N. N., 2017. Tax, financial and social regulatory mechanisms within the knowledge-driven Economy. Blockchain algorithms and fog computing for the efficient regulation, in 2017 XX IEEE International Conference on Soft Computing and Measurements (SCM) (pp. 709-712), St. Petersburg.

Risius, M. and Spohrer, K., 2017. A blockchain research framework What we (don't) know, where we go from here, and how we will get there. Business \& Information Systems Engineering, 59(6), 385-409. doi: 10.1007/s12599-017-0506-0.

Rosic, A., n.d. What is hashing? [Step-by-step guide-under hood of blockchain], from https://blockgeeks.com/guides/what-is-hashing/, accessed 10 May 2020.

Rozario, A. M. and Vasarhelyi, M. A., 2018. Auditing with smart contracts, International Journal of Digital Accounting Research, 18, 1-27. doi: 10.4192/1577-8517-v18_1.

Rückeshäuser, N., 2017. Do we really want blockchain-based accounting? Decentralized consensus as enabler of management override of internal Controls, in Wirtschaftsinformatik 2017 Proceedings (pp. 16-30). doi: 10.1016/S01674048(97)90261-3.

Saberi, S., Kouhizadeh, M., Sarkis, J. and Shen, L. M., 2019. Blockchain technology and its relationships to sustainable supply chain management, International Journal of Production Research 57(7), 2117-2135. doi: 10.1080/00207543.2018.1533261.

Satoh, A. and Inoue, T., 2007. ASIC-hardware-focused comparison for hash functions MD5, RIPEMD-160, and SHS. Integration, 40(1), 3-10. https://doi.org/10.1016/j.vlsi.2005.12.006

Schmitz, J. and Leoni, G., 2019. Accounting and auditing at the time of blockchain technology: A research agenda, Australian Accounting Review, 29(2), 331-342. doi: 10.1111/auar.12286.

Schwab, K., 2016. The fourth industrial revolution: what it means, how to respond, World Economic Forum, from https://www.weforum.org/agenda/2016/01/the-fourthindustrial-revolution-what-it-means-and-how-to-respond, accessed 19 February 2020.

Schwanke, A., 2017. Bridging the digital gap: How tax fits into cryptocurrencies and blockchain development, International Tax Review, 28(3), 20-25.

Shim, Y. and Shin, D. H., 2016. Analyzing China's fintech industry from the perspective of actor-network theory, Telecommunications Policy, 40(2-3), 168-181. doi: 10.1016/j.telpol.2015.11.005.

Shipman, J., 2016. Infographic: Blockchain explained, from https://www.digitalpulse.pwc.com.au/pwc-blockchain-infographic/, accessed 10 May 2020.

Sikka, P., 2010. Smoke and mirrors: Corporate social responsibility and tax avoidance. Accounting Forum, 34(3), 153-168.

Singh, H., Jain, G., Munjal, A. and Rakesh, S., 2019. Blockchain technology in corporate governance: disrupting chain reaction or not?, Corporate Governance, 20(1), 67-86. doi: 10.1108/CG-07-2018-0261. 
Šmejkal, V., 2015. Competition law and the social market economy goal of the EU. International Comparative Jurisprudence, 1(1), 33-43.

Smith, K. J. and Dhillon, G., 2019. Assessing blockchain potential for improving the cybersecurity of financial transactions, Managerial Finance. doi: 10.1108/MF-06-20190314.

Smith, R., 2018. What is delegated proof of stake? | Exploring the consensus algorithm, from https://coincentral.com/what-is-delegated-proof-of-stake-exploring-the-consensusalgorithm/, accessed 10 May 2020.

Tandulwadikar, A., 2016. Blockchain in banking: A measured approach, Cognizant Reports, from https://www.cognizant.com/whitepapers/Blockchain-in-Banking-A-MeasuredApproach-codex 1809.pdf, accessed 1 May 2020.

Tapscott, A. and Tapscott, D., 2017. How blockchain is changing finance, Harvard Business Review, 1(9) 2-5.

The Telegraph, 2019. London Stock Exchange collaborates on first issue of blockchain 'token' shares, from https://www.telegraph.co.uk/technology/2019/04/15/london-stockexchange-accepts-first-listing-blockchain-token/, accessed 26 April 2020.

Truby, J., 2018. Decarbonizing bitcoin: Law and policy choices for reducing the energy consumption of blockchain technologies and digital currencies, Energy Research \& Social Science, 44, 399-410.

Ulyanava, K., 2018. Legal regulation of the crypto-currency taxation, Open Journal for Legal Studies, 1(1), 1-8.

Vaidya, K., 2016. Decoding the enigma of bitcoin mining — Part I : Mechanism, from https://medium.com/all-things-ledger/decoding-the-enigma-of-bitcoin-mining-

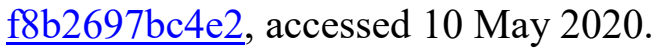

Vaitkune, S., 2017. The most comprehensive cryptocurrency wallet guide, from https://medium.com/@,fastinvest/the-most-comprehensive-cryptocurrency-walletguide-5e820a26ed44, accessed 10 May 2020.

van Rijswijk, L., Hermsen, H. and Arendsen, R., 2018. Exploring the future of taxation: A blockchain scenario study, paper presented at the 6th annual Tax Administration Research Centre (TARC) Workshop, 23-24 April, Exeter, UK.

Venit, J. S., 1996. EU competition law - enforcement and compliance: An overview. Antitrust Law Journal, 65(1), 81-104.

Vishnevsky V. P. and Chekina V. D., 2018. Robot vs. tax inspector or how the fourth industrial revolution will change the tax system: A review of problems and solutions, Journal of Tax Reform, 4(1), 6-26.

Wang, Y., Singgih, M., Wang, J. and Rit, M., 2019. Making sense of blockchain technology: How will it transform supply chains?, International Journal of Production Economics, $211,221-236$.

Wang, H., Ma, S., Dai, H.-N., Imran, M. and Wang, T., 2020. Blockchain-based data privacy management with nudge theory in open banking, Future Generation Computer Systems, $110,812-823$.

Warnez, J., 2017. Revenue registration and automatic taxation for platform businesses on blockchain, MSc Dissertation, Copenhagen Business School. 
Webb, N., 2018. A fork in the blockchain: Income tax and the bitcoin/bitcoin cash hard fork, North Carolina Journal of Law \& Technology, 19(4), 283-311.

Wijaya D. A., Liu, J. K., Steinfeld, R., Liu, D., Junis, F. and Suwarsono, D. A., 2019. Designing smart contract for electronic document taxation, in Mu Y., Deng, R. and Huang X (eds), Cryptology and Network Security, CANS 2019, Lecture Notes in Computer Science, Volume 11829 (pp. 199-213), Cham, Springer.

Wijaya, D. A., Liu, J. K., Suwarsono, D. A. and Zhang, P., 2017. A new blockchain-based Value-Added Tax system, in Okamoto, T., Yu, Y., Au, M. H. and Li, Y. (eds), Provable Security (pp. 471-486), USA, Springer.

Witzig, P. and Salomon, V., 2018. Cutting out the middleman: A case study of blockchaininduced reconfigurations in the Swiss Financial Services Industry, working paper 1 2018/e, The Circulation of Wealth, Université de Neuchâtel.

Wonglimpiyarat, J., 2018. Challenges and dynamics of fintech crowd funding: An innovation system approach, Journal of High Technology Management Research, 29(1), 98-108. doi: 10.1016/j.hitech.2018.04.009.

World Economic Forum, 2015. Deep shift: Technology tipping points and societal impact, from

http://www3.weforum.org/docs/WEF GAC15 Technological Tipping Points report 2015.pdf\#page $=24$, accessed 15 April 2020.

World Economic Forum, 2016. All you need to know about blockchain, explained simply, from https://www.weforum.org/agenda/2016/06/blockchain-explained-simply/, accessed 15 April 2020.

World Trade Organization (WTO)., 2015a. Trade and Tariffs, Geneva, WTO.

World Trade Organization (WTO), 2015b. World Trade Report 2015: Speeding up Trade Benefits and Challenges of Implementing the WTO Trade Facilitation Agreement, Geneva, WTO.

Wouda, H. P. and Opdenakker, R., 2019. Blockchain technology in commercial real estate transactions, Journal of Property Investment and Finance, 37(6), pp. 570-579. doi: 10.1108/JPIF-06-2019-0085.

Wright, A. and De Filippi, P., 2015. Decentralized blockchain technology and the rise of Lex Cryptographia (10 March 2015), from SSRN: https://ssrn.com/abstract=2580664 or doi: $10.2139 /$ ssrn.2580664.

Yermack, D., 2017. Corporate governance and blockchains, Review of Finance, 21(1), 7-31.

Yew, L. K., 2018. Blockchain: Opportunities and challenges for taxation, from https://scholarbank.nus.edu.sg, accessed 4 May 2020.

Yli-Huumo, J., Ko, D., Choi, S., Park, S. and Smolander, K., 2016. Where is current research on blockchain technology? - a systematic review, PLOS ONE, 11(10) e0163477. doi 10.1371/journal.pone.0163477.

Zheng, Z., Xie, S., Dai, H., Chen, X. and Wang, H., 2017. An overview of blockchain technology: Architecture, consensus, and future trends, in 2017 IEEE International Congress on Big Data (BigData Congress), Honolulu, HI (pp. 557-564). doi: 10.1109/BigDataCongress.2017.85. 
Zhu, H. and Zhou, Z. Z., 2016. Analysis and outlook of applications of blockchain technology to equity crowdfunding in China, Financial Innovation, 2, Article 29. doi: 10.1186/s40854-016-0044-7. 